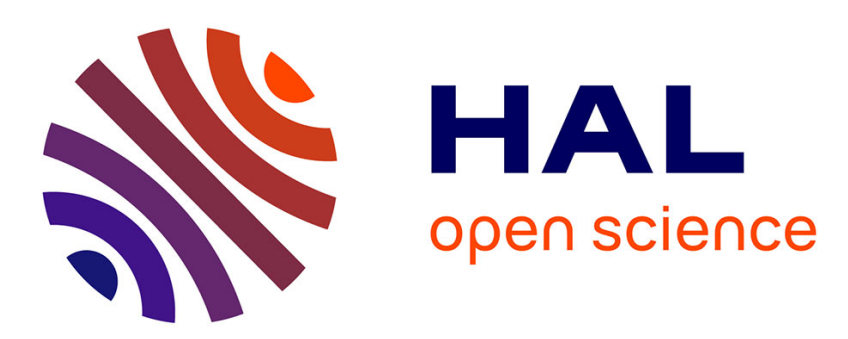

\title{
Numerical solution of the viscous flows in a network of thin tubes: equations on the graph
}

Éric Canon, Frédéric Chardard, Grigory Panasenko, Olga Štikonienẻ

\section{To cite this version:}

Éric Canon, Frédéric Chardard, Grigory Panasenko, Olga Štikonienė. Numerical solution of the viscous flows in a network of thin tubes: equations on the graph. Journal of Computational Physics, 2021, 435, pp.110262. 10.1016/j.jcp.2021.110262 . hal-02407080

\section{HAL Id: hal-02407080 \\ https://hal.science/hal-02407080}

Submitted on 12 Dec 2019

HAL is a multi-disciplinary open access archive for the deposit and dissemination of scientific research documents, whether they are published or not. The documents may come from teaching and research institutions in France or abroad, or from public or private research centers.
L'archive ouverte pluridisciplinaire HAL, est destinée au dépôt et à la diffusion de documents scientifiques de niveau recherche, publiés ou non, émanant des établissements d'enseignement et de recherche français ou étrangers, des laboratoires publics ou privés. 


\title{
Numerical solution of the viscous flows in a network of thin tubes: equations on the graph
}

\author{
Éric Canon ${ }^{a}$, Frédéric Chardard $^{a}$, Grigory Panasenko $^{a, b}$, Olga Štikoniené ${ }^{b}$
}

${ }^{a}$ Univ Lyon, UJM-Saint-Étienne, CNRS, Institute Camille Jordan UMR 5208, SFR MODMAD FED 4169, F-42023, SAINT-ÉTIENNE, FRANCE

${ }^{b}$ Institute of Applied Mathematics, Vilnius University, Naugarduko 24, VILNIUS, LITHUANIA

\begin{abstract}
A non-stationary flow in a network of thin tubes is considered. Its one-dimensional approximation was proposed in a paper by G.Panasenko and K.Pileckas, Flows in a tube structure: equation on the graph, JMP (2014). It consists of a set of equations with weakly singular kernels, on a graph, for the macroscopic pressure. A new difference scheme for this problem is proposed. Several variants are discussed. Stability and convergence are carefully investigated, theoretically and numerically. In addition, numerical results are compared to the direct numerical solution of the full dimension Navier-Stokes equations.
\end{abstract}

Key Words: Navier-Stokes Equations, Asymptotic models, weakly singular kernels, multi-scale problems.

AMS classification: 78M35, 74G10, 35Q30, 35R09, 34K28. 


\section{Contents}

1 Introduction 2

1.1 Thin tubes structure $\ldots \ldots \ldots \ldots \ldots \ldots \ldots$

1.2 Numerical scheme and convergence $\ldots \ldots \ldots \ldots \ldots \ldots$

\begin{tabular}{|lll}
2 & Continuous problem on the graph & $\mathbf{7}$
\end{tabular}

2.1 Vector spaces and notations $\ldots \ldots \ldots \ldots \ldots \ldots$. . . . . . . . . . . . 7

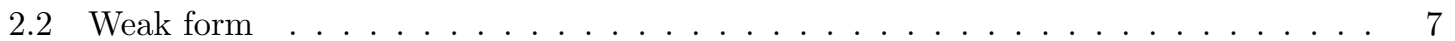

2.3 Regularity of the solution $\ldots \ldots \ldots \ldots \ldots \ldots$

$2.3 .1 \quad$ Regularity with respect to the space variable $\ldots \ldots \ldots \ldots$. . . . . . . 8

$2.3 .2 \quad$ Regularity with respect to the time variable $\ldots \ldots \ldots \ldots$. . . . . . . 8

\begin{tabular}{lll}
\hline & Galerkin method & 8
\end{tabular}

3.1 Time discretization . . . . . . . . . . . . . . . . . . . . . . 9

3.2 Continuity and coercivity of the approximate bilinear form . . . . . . . . . . . 9

3.3 Total discretization on the graph $\ldots \ldots \ldots \ldots \ldots$

3.4 Discretization in the cross-section . . . . . . . . . . . . . . . . . . . . 14

3.4 .1 Exact kernels . . . . . . . . . . . . . . . . . . . . . . . . . . . . 14

3.4 .2 Numerical approximation of the kernels . . . . . . . . . . . . . . . . . 15

3.4.3 Corrected approximation $\ldots \ldots \ldots \ldots \ldots \ldots$

3.5 Remarks on the solving of the scheme $\ldots \ldots \ldots \ldots \ldots \ldots$

3.5.1 Using the block triangular structure . . . . . . . . . . . . . . . . . . 16

3.5 .2 Using the Fast Fourier Transform $\ldots \ldots \ldots \ldots$. . . . . . . . . . 16

3.5.3 Coupling with the cross-section $\ldots \ldots \ldots \ldots \ldots$. . . . . . . . . . 17

$\begin{array}{|lr|}4 \text { Convergence of the scheme } & 18\end{array}$

$\begin{array}{lll}5 & \text { Numerical results } & 23\end{array}$

5.1 Numerical accuracy $\ldots \ldots \ldots \ldots \ldots \ldots \ldots \ldots$. . . . . . . . . . . . . . . . . 23

5.1 .1 Test case on a single tube . . . . . . . . . . . . . . . . . 23

$5.1 .2 \quad$ Numerical accuracy when $d=2 \ldots \ldots \ldots \ldots \ldots . \ldots \ldots 24$

$5.1 .3 \quad$ Numerical accuracy when $d=3$. . . . . . . . . . . . . . . . . . . . 25

5.2 Comparison with Navier-Stokes equations _ . . . . . . . . . . . . . . . 25

5.2 .1 Geometry and boundary condtions . . . . . . . . . . . . . . 25

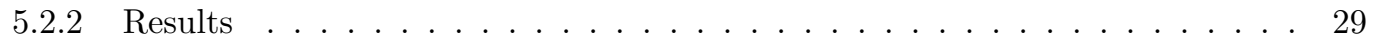

\section{Introduction}

Modeling and effective computations of viscous flows in networks of thin pipes are important topics, notably in microfluidics and flows in blood vessels. Because of the complexity of the geometry of such $2 \mathrm{D}$ or $3 \mathrm{D}$ networks, it is important to have simplified, but relevant, models for these flows, and then efficient schemes for solving them numerically. The main aim of the present work is to design and study numerical schemes for such a simplified model which was obtained by Panasenko \& Pileckas, and consists in a time dependent, non local in time diffusion equations for the pressure, set on a 1D-graph, with appropriate (Kirchhoff) junction conditions on the vertices of the graph. It is described in [19] and mathematically justified in [21, by letting the diameters of the pipes tend to zero, with appropriate scalings on the data. Also, we compare numerically the original multidimensional problem with its simplified version, in order to establish the relevance of this limit model [19.

Let us go first more into detail about this modeling. Stationary laminar incompressible viscous flows in a thin tube were first studied empirically [25] and theoretically [7] in the nineteenth century. They were described by the stationary Poiseuille profile. However, the time scale of the phenomena may prevent stationary approximation to be valid as noticed by Womersley 32 for blood flows. In that case, one needs to use a time-dependent Poiseuille type profile: a Womersley profile. Pileckas et al. [24, 10, 18] studied the behaviour of the incompressible Navier-Stokes equations in an infinite tube 
when the diameter of the pipe is small, proving the existence and relevance of the time-dependent Poiseuille profile in a quite general setting. Panasenko \& Pileckas studied the incompressible NavierStokes equation in a network of thin tubes, when the flow is stationary [16, 17, 20, 22, or timedependent [20, 21. In that case of non-steady Navier-Stokes equations in the tube-structure, similarly to $24,10,18$, they obtain $([19,21])$ a limit, simplified model retaining this Womersley effect. The model consists in a one dimensional, nonlocal in time, diffusion equation on the 1Dskeleton (or graph) of the initial structure for the pressure, coupled with heat equations set in rescaled cross sections of the tubes of this initial structure, with Kirchhoff type conditions at the vertices of the graph and the continuity condition for the pressure.

The main aim of the present paper is to propose and study a numerical scheme, with several variants, for this model. We show convergence and stability without any special restriction on the shape of the cross sections of the tubes. An important point in designing the schemes is that in the coupling mentioned above the heat equations in the cross sections can be solved explicitly with respect of the gradient of the pressure, allowing to rewrite the equations for the pressure on the edges as diffusion equations with convolution in time. In designing the schemes, we have to pay a particular attention on how to discretize this couplings, or convolution products. Different possibilities are investigated : exact kernels for special geometry of the cross-sections, numerical approximation on the kernels for general cases, with order 1 or 2 in time, with of without corrections for small times, using asymptotics for small times obtained in the second part of this work [8].

At this point, let us underline that this work is in two parts: properties of the kernels are investigated in detail in a forthcoming paper ([8]). However, as mentioned above, some of these properties are used in the numerics of the present paper.

Last, we also check the convergence numerically and compare the asymptotic model with the directly numerically solved Navier-Stokes equations.

Let us mention well-known alternative models for network of pipes, considered for instance in 1, 6, 9, 11, 28, 29, 31. These models are hyperbolic on the graph and are designed for a different context (high Reynolds number, elastic walls, as in arteries) than ours (modest Reynolds number, rigid walls as in arterioles). See [19, 21] and the just mentioned references for detail.

Now, the plan of the paper is as follows. In Section, we recall the original and limit models in [19, 21] and give a brief presentation of our scheme. In Section we present a few notations and the weak form of the equations on the graph and prove a few additional regularity results that are needed in this paper. In Section 3 we go more into detail about the discretization: the scheme as presented in Section 1 is derived from the weak form as a Galerkin method; we show coercivity (and continuity) of that approximate bilinear form; we give a few words on the discretization in the cross sections (the details been given in the second part of this work [8]); and last some remarks on the implementation of the scheme. Section 4 is devoted to the main results : convergences of the scheme and rates of convergence, in relation with the accuracy on the approximation of the kernels: Theorems 1 and 2 and Corollary 1. Section 5 is devoted to the presentation of numerical results : comparison with exact test cases and comparisons with full 3D Navier-Stokes equations.

\subsection{Thin tubes structure}

We first describe a $d$-dimensional thin tubes structure, where $d \in\{2,3\}$. Let $O_{1}, \ldots O_{N}$ be different vertices of $\mathbb{R}^{d}, e_{1}, \ldots, e_{M}$ closed segments $e_{j}=\left[O_{i_{j}} O_{k_{j}}\right]$ connecting these vertices. We assume that these edges can only intersect at vertices. The end points of the skeleton of the graph, that is the vertices that belong to a single edge are denoted by $O_{1}, \ldots, O_{N_{1}}\left(0 \leq N_{1} \leq N-1\right)$ while $O_{N_{1}+1}, \ldots, O_{N}$ corresponds to junctions between the pipes. We fix a positive orientation for all edges $e_{j}=\left[O_{i_{j}} O_{k_{j}}\right]$ as the direction from $O_{i_{j}}$ to $O_{k_{j}}$. For simplicity, we use the same notation $e_{j}$ for the vector $\overrightarrow{O_{i_{j}} O_{k_{j}}}$ as for the edge.

Let $\sigma_{1}, \ldots, \sigma_{M}$ be smooth domains of $\mathbb{R}^{d-1}$ representing the cross-sections of the pipes. The variable in the cross-section direction is denoted with a hat: $\hat{x} \in \mathbb{R}^{d-1}$. Let $R^{\left(e_{1}\right)}, \ldots, R^{\left(e_{M}\right)}$ be rotations in $\mathbb{R}^{d}$ such that:

$$
R^{\left(e_{j}\right)}\left(\hat{0},\left|e_{j}\right|\right)=e_{j}
$$




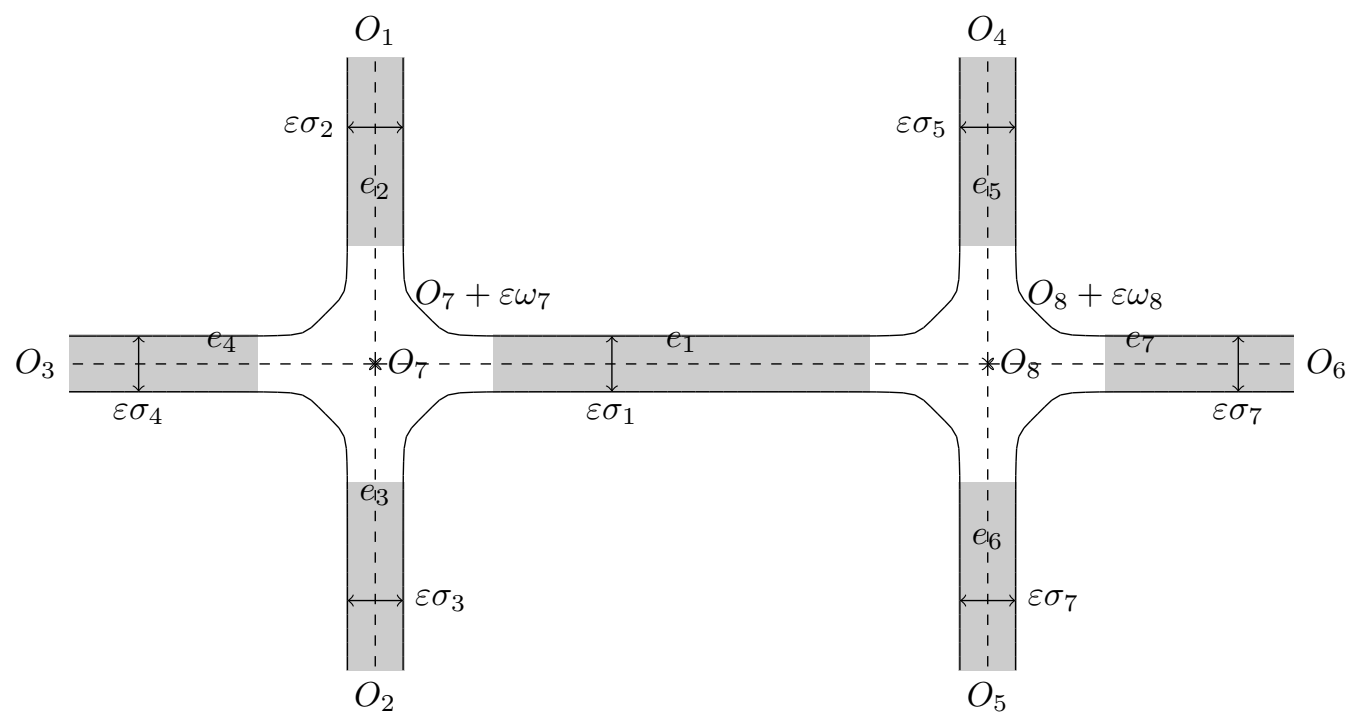

Figure 1: Example of thin structure when $d=2, N_{1}=6, N=8, M=7$.

Let $\omega_{N_{1}+1}, \ldots, \omega_{M}$ be bounded domains of $\mathbb{R}^{d}$ containing 0 which represent the junctions of the tubes and define the initial d-dimensional network of tubes as :

$$
\Omega^{\varepsilon}=\left(\bigcup_{i=N_{1}+1}^{N}\left(O_{i}+\varepsilon \omega_{i}\right)\right) \bigcup\left(\bigcup_{j=1}^{N} O_{i_{j}}+R^{\left(e_{j}\right)}\left(\varepsilon \sigma_{j} \times\right] 0,\left|e_{j}\right|[)\right) .
$$

We assume that, for $\varepsilon$ small enough, $\Omega^{\varepsilon}$ is connected, smooth except at the end sections $\bigcup_{j=1}^{N_{1}} R^{\left(e_{j}\right)}\left(\varepsilon \partial \sigma_{j} \times\right.$ $\{0\})$. Last,

$$
\mathcal{B}=\bigcup_{j=1}^{M} e_{j}
$$

is the graph of the structure.

We now, states the equations of the model we are dealing with. These equations are set on the graph $\mathcal{B}$ and were obtained in 21] by passing to the limit as $\varepsilon$ tends to 0 on the following incompressible Navier-Stokes equations with Dirichlet's boundary conditions, in $\Omega^{\varepsilon}$ :

$$
\begin{cases}\frac{\partial u^{\varepsilon}}{\partial t}+\left(u^{\varepsilon} \cdot \nabla u^{\varepsilon}\right)-\Delta u^{\varepsilon}=-\nabla p^{\varepsilon} & \text { on } \Omega^{\varepsilon}, \\ \nabla \cdot u^{\varepsilon}=0 & \text { on } \Omega^{\varepsilon}, \\ u^{\varepsilon}\left(O_{i}+R^{\left(e_{j}\right)}(\hat{x}, 0), t\right)=R^{\left(e_{j}\right)}\left(g^{i}\left(\varepsilon^{-1} \hat{x}, \varepsilon^{-2} t\right)\right) & \text { on } O_{i}+R^{\left(e_{j}\right)}\left(\varepsilon \sigma_{j} \times\{0\}\right), i=1, \ldots, N_{1}, O_{i} \in e_{j}, \\ u^{\varepsilon}=0 & \text { on } \partial \Omega^{\varepsilon} \backslash\left(\bigcup_{i=1}^{N_{1}}\left(O_{i}+\left.R^{\left(e_{j}\right)}\left(\varepsilon \sigma_{j} \times\{0\}\right)\right|_{O_{i} \in e_{j}}\right)\right), \\ \left.u^{\varepsilon}\right|_{t=0}=0, & \\ p^{\varepsilon}\left(O_{1}, t\right)=0 . & \end{cases}
$$

Here, $g^{i}: \sigma_{i} \times \mathbb{R}^{+} \rightarrow \mathbb{R}^{d}$ denotes the velocity profile in a reference frame at the free extremity of the tube $i$ for $i \in\left\{1, \ldots, N_{1}\right\}$. Let $\frac{\partial}{\partial x^{\left(e_{j}\right)}}$ denote the derivative along the vector $\left|e_{j}\right|^{-1} e_{j}$. According to [20, 21], under some hypotheses on $g^{i}, p^{\varepsilon}\left(x, \varepsilon^{2} t\right)$ can be approximated at the leading order by 
$P(x, t)$ where $P$ is the solution to the following problem on $\mathcal{B}$ :

$$
\begin{cases}-\frac{\partial}{\partial x^{\left(e_{j}\right)}} L^{\left(\sigma_{j}\right)} \frac{\partial P}{\partial x^{\left(e_{j}\right)}}(x, t)=F(x, t) & \text { for } x \in e_{j}, \\ \sum_{O_{i} \in e_{j}} \alpha_{i, j} L^{\left(\sigma_{j}\right)} \frac{\partial P}{\partial x^{\left(e_{j}\right)}}\left(O_{i}, t\right)=-\Psi_{i}(t) \quad \text { for } i=1, \ldots, N, \\ P \text { is continuous on the graph, } \\ P\left(O_{1}, t\right)=0 .\end{cases}
$$

In $1.22_{2}$ : the summation is over all edges $e_{j}$ having $O_{i}$ as an end point; $\alpha_{i, j}=1$ if the orientation $e_{j}$ starting from $O_{i}$ is positive, and $\alpha_{i, j}=-1$ otherwise. The right hand sides in $(1.2)_{1}$ are given by :

$$
\Psi_{i}(t)= \begin{cases}\int_{\sigma_{j}}\left\langle g^{i}(\hat{x}, t),\left(\hat{0}, \alpha_{i, j}\right)\right\rangle \mathrm{d} \hat{x} & \text { if } i \in\left\{1, \ldots, N_{1}\right\}, O_{i} \in e_{j} \\ 0 & \text { if } i \in\left\{N_{1}+1, \ldots, N\right\}\end{cases}
$$

where $\langle\cdot, \cdot\rangle$ denotes the Euclidean product.

The operator $L^{\left(\sigma_{j}\right)}$ are nonlocal in time operators relating the macroscopic equation for the pressure 1.2 to the microscopic structures $\sigma_{j}$. For each such cross section $\sigma$ they are defined: $L^{(\sigma)}$ : $L^{2}(0,+\infty) \rightarrow H_{0}^{1}(0,+\infty)$ by:

$$
\forall t>0,\left(L^{(\sigma)} q\right)(t)=\int_{\sigma} U(\hat{x}, t) \mathrm{d} \hat{x}
$$

where $U$ is solution of

$$
\begin{cases}\frac{\partial U}{\partial t}-\Delta_{\hat{x}} U=q(t) & \text { on } \sigma \\ U=0 & \text { on } \partial \sigma \\ U=0 \text { if } t=0 & \text { on } \sigma .\end{cases}
$$

\section{Remarks on (1.2).}

(i) In usual applications $F=0$, but it is useful, at least for building test problems with exact solutions, to consider more general $F$.

(ii) Equation $(1.2)_{2}$ is the Kirchhoff condition which expresses the conservation of mass at the junctions and at the ends of the graph. If the system has a solution, it satisfies the following compatibility condition:

$$
\forall t \in[0, T], \quad \sum_{\ell=1}^{N} \Psi_{\ell}(t)+\int_{\mathcal{B}} F(x, t) \mathrm{d} x=0 .
$$

where $\int_{\mathcal{B}}$ denotes the integral over all edges of the graph (see 2.13). If this compatibility condition is satisfied then, the equation on $\Psi_{1}$ is redundant and can be omitted in the discretization.

(iii) Pressure can be obtained by solving system $(1.2)$ and then, the velocity profile at some distance from the junctions by solving (1.4). This is discussed in Section 3.5.3. To obtain the next order of approximation, the continuity of pressure 1.2$]_{3}$ may be replaced by a coupling with Navier-Stokes equations in regions surrounding the junctions (see [21]). Such couplings are also discussed and numerically tested in [3, 4, 5. 


\subsection{Numerical scheme and convergence}

The main goal of this article is to propose a numerical method to solve problem (1.2). This is done in the present section.

First, the operator $L^{(\sigma)}$ can be rewritten as a convolution operator:

$$
\left(L^{(\sigma)} q\right)(t)=\int_{0}^{t} K^{(\sigma)}(t-\tau) q(\tau) d \tau,
$$

where the kernel $K^{(\sigma)}$ is defined by

$$
K^{(\sigma)}(t)=\int_{\sigma} V(\hat{x}, t) \mathrm{d} \hat{x}
$$

$V$ being the solution of the heat equation:

$$
\begin{cases}\frac{\partial V}{\partial t}-\Delta V=0 & \text { on } \sigma \times] 0,+\infty[ \\ V(., 0)=1 & \text { on } \sigma, \\ V(\hat{x}, t)=0 & t>0, \hat{x} \in \partial \sigma .\end{cases}
$$

The discretization is then as follows. Let $\left(t_{0}=0, t_{1}=k, \ldots, t_{Q}=k Q=T\right)$ be a subdivision of $[0, T]$ with a uniform step $k$. For each edge $e$, we introduce a subdivision $\left(x_{0}^{(e)}=0, x_{1}^{(e)}=h^{(e)}, \ldots, x_{S^{(e)}}^{(e)}=\right.$ $\left.S^{(e)} h^{(e)}=|e|\right)$ of $[0,|e|]$ with a uniform step $h^{(e)}$. Let $X_{s}^{\left(e_{i}\right)}=O_{i}+R^{(e)}\left(\hat{0}, x_{s}^{(e)}\right)$.

Let $P_{s, q}^{(e)}$ be an approximation of $P\left(X_{s}^{(e)}, t_{q+1 / 2}\right)$, where $t_{q+1 / 2}=\frac{t_{q}+t_{q+1}}{2}$. Consider the following difference scheme:

$$
\begin{aligned}
& f_{s, q+1}^{\left(e_{j}\right)}=-k \sum_{\tilde{q}=0}^{q} \tilde{K}_{q-\tilde{q}}^{\left(\sigma_{j}\right)} \frac{P_{s+1, \tilde{q}}^{\left(e_{j}\right)}-2 P_{s, \tilde{q}}^{\left(e_{j}\right)}+P_{s-1, \tilde{q}}^{\left(e_{j}\right)}}{\left(h^{\left(e_{j}\right)}\right)^{2}} \text { if }\left\{\begin{array}{l}
1 \leq j \leq M, \\
0<s<S^{\left(e_{j}\right)} \\
0 \leq q<Q
\end{array},\right. \\
& \Psi_{\ell, q+1}=\sum_{1 \leq j \leq M, O_{\ell} \in e_{j}}\left(-\frac{h^{\left(e_{j}\right)}}{2} f_{s, \tilde{q}+1}^{\left(e_{j}\right)}-k \sum_{\tilde{q}=0}^{q} \tilde{K}_{q-\tilde{q}}^{\left(\sigma_{j}\right)} \frac{P_{s+\alpha_{\ell, j}, \tilde{q}}^{\left(e_{j}\right)} P_{s, \tilde{q}}^{\left(e_{j}\right)}}{h^{\left(e_{j}\right)}}\right) \text { if }\left\{\begin{array}{l}
2 \leq \ell \leq N \\
0 \leq q<Q
\end{array},\right. \\
& P_{s, q}^{(e)}=P_{\tilde{s}, q}^{(\tilde{e})} \text { if } e, \tilde{e} \in\left\{e_{1}, \ldots, e_{M}\right\}, s \in\left\{0, S^{(e)}\right\}, \tilde{s} \in\left\{0, S^{(\tilde{e})}\right\}, X_{s}^{(e)}=X_{\tilde{s}}^{(\tilde{e})}, 0 \leq q<Q \\
& P_{0, q}^{(e)}= 0 \text { if } X_{0}^{(e)}=O_{1}, 0 \leq q<Q,
\end{aligned}
$$

where:

- $\tilde{K}_{q}^{(\sigma)}$ is either $K_{q}^{(\sigma)}=\frac{1}{k} \int_{t_{q}}^{t_{q+1}} K^{(\sigma)}(t) \mathrm{d} t$ for $q \geq 0$ when the kernel can be computed explicitly (see 3.22-3.25) or a suitable approximation of it (see Section 3.4.

- $\Psi_{\ell, q}=\Psi_{\ell}\left(t_{q}\right)$ for $0 \leq q \leq Q$ and $1 \leq \ell \leq N$,

- $f_{s, q}^{\left(e_{j}\right)}=F\left(X_{s}^{\left(e_{j}\right)}, t_{q}\right)$.

- in $1.9, s=0, \alpha_{\ell, j}=1$ if $X_{0}^{\left(e_{j}\right)}=O_{\ell}$ and $s=S^{\left(e_{j}\right)}, \alpha_{\ell, j}=-1$ if $X_{S^{\left(e_{j}\right)}}^{\left(e_{j}\right)}=O_{\ell}$. Note that $X_{s+\alpha_{\ell, j}}^{\left(e_{j}\right)}$ is the node closest to $O_{\ell}$ among those on the edge $e_{j}$.

A similar numerical scheme can be found in [15, 26, 27, when $\mathcal{B}$ is a tree, the cross-section a disc, and all the data are periodic in time. However, the convergence is not addressed in [15, 26, 27.

Let:

$$
\theta(k)=\max _{1 \leq j \leq M}\left|K_{0}^{\left(\sigma_{j}\right)}-\tilde{K}_{0}^{\left(\sigma_{j}\right)}\right|+\sum_{q=1}^{Q-1}\left|K_{q}^{\left(\sigma_{j}\right)}-K_{q-1}^{\left(\sigma_{j}\right)}-\tilde{K}_{q}^{\left(\sigma_{j}\right)}+\tilde{K}_{q-1}^{\left(\sigma_{j}\right)}\right| .
$$

Note that $\theta(k)=0$ if $\tilde{K}_{q}^{(\sigma)}=K_{q}^{(\sigma)}$. 
In Section 3 below, we first reformulate this scheme as a Galerkin method. In Propositions 1 and 2 of Section 3.2 , we then give two different sufficient stability conditions on $\tilde{K}_{q}^{(\sigma)}$, which are verified when $\theta(k)=0$.

Last in Section 4, we prove our main result: the scheme is convergent if $\theta(k) \rightarrow 0$ and is of $L^{2}$ order 1 in time and $H^{1}$-order 1 in space if $\theta(k)=0$. This is Theorem 1 . Furthermore, with additional regularity assumptions, these orders of convergence can be improved. This is Theorem 2 and Corollary 1.

\section{Continuous problem on the graph}

In this section, we get results on the continuous problem 1.2 that are used in the proof of Theorem 1.

\subsection{Vector spaces and notations}

To avoid summations, let us denote:

$$
\int_{\mathcal{B}} f(x) \mathrm{d} x=\sum_{j=1}^{M} \int_{0}^{\left|e_{j}\right|} f\left(O_{i}+R_{j}\left(\hat{0}, x_{d}\right)\right) \mathrm{d} x_{d} .
$$

The operators $L^{\left(\sigma_{j}\right)}$ and $\frac{\partial}{\partial x^{\left(e_{j}\right)}}$ will be applied to functions which are defined on $\mathcal{B}$. To shorten notations and to be able to use the previous notation, let us denote $\bar{\sigma}(x)=\sigma_{j}$ and $\bar{e}(x)=e_{j}$ when $x \in e_{j}$ and $x$ is not a vertex, so that $\frac{\partial f}{\partial x^{(\bar{e})}}(x)=\frac{\partial f}{\partial x^{\left(e_{j}\right)}}(x)$ and $L^{\left(\sigma_{j}\right)} g(x, t)=L^{(\bar{\sigma})} g(x, t)$.

Define the norms and the vector spaces:

$$
\begin{gathered}
\|f\|_{L^{2}(B)}^{2}=\int_{\mathcal{B}}|f|^{2} \mathrm{~d} x, \\
\|f\|_{H_{d c}^{k}(\mathcal{B})}^{2}=\int_{\mathcal{B}}|f|^{2}+\left|\frac{\partial f}{\partial x^{(\bar{e})}}\right|^{2}+\ldots+\left|\frac{\partial^{k} f}{\left(\partial x^{(\bar{e})}\right)^{k}}\right| \mathrm{d} x, \\
L^{2}(\mathcal{B})=\left\{f: \mathcal{B} \rightarrow \mathbb{R} \mid\|f\|_{L^{2}(\mathcal{B})}<+\infty\right\}, \\
H^{1}(\mathcal{B})=\left\{f: \mathcal{B} \rightarrow \mathbb{R} \mid f\left(O_{1}\right)=0, f \text { continuous, }\|f\|_{H_{d c}^{1}(\mathcal{B})}<+\infty\right\}, \\
\forall k \in \mathbb{N}^{*}, H_{d c}^{k}(\mathcal{B})=\left\{f: \mathcal{B} \rightarrow \mathbb{R} \mid\|f\|_{H_{d c}^{k}(\mathcal{B})}^{2}<+\infty\right\} .
\end{gathered}
$$

Notice that a function of $H_{d c}^{k}(\mathcal{B})$ may be discontinuous at the junctions $O_{1}, O_{2}, \ldots, O_{\ell}$.

For any Banach space $E$ and $r \in \mathbb{N}^{*}$, let us define:

$$
\begin{gathered}
H^{r}(0, T, E)=\left\{f:[0, T] \rightarrow E \mid \int_{0}^{T}\left(|f(t)|_{E}^{2}+\left|f^{\prime}(t)\right|_{E}^{2}+\ldots+\left|f^{(r)}(t)\right|_{E}^{2}\right) \mathrm{d} t<+\infty\right\}, \\
H_{00}^{r}(0, T, E)=\left\{f \in H^{r}(0, T, E) \mid f(0)=0, \ldots, f^{(r-1)}(0)=0\right\} \\
H^{r}(0, T)=H^{r}(0, T, \mathbb{R}), H_{00}^{r}(0, T)=H_{00}^{r}(0, T, \mathbb{R}) .
\end{gathered}
$$

\subsection{Weak form}

Let us assume that $F \in H_{00}^{1}\left(0, T, L^{2}(\mathcal{B})\right)$ and that $\Psi_{1}, \ldots, \Psi_{N} \in H_{00}^{1}(0, T)$ and satisfy the compatibility condition 1.5.

The well-posedness in $L^{2}\left(0, T, H^{1}(\mathcal{B})\right)$ of system 1.2 was proved by means of the Lax-Milgram lemma in [20, 21]. The weak form of 1.2 is as follows: find $P \in L^{2}\left(0, T, H^{1}(\mathcal{B})\right)$ such that:

$$
\forall \psi \in L^{2}\left(0, T, H^{1}(\mathcal{B})\right), \quad a_{T}(P, \psi)=b_{T}(\psi)
$$


where

$$
\begin{gathered}
a_{T}(P, \psi)=\int_{0}^{T} \int_{\mathcal{B}} \frac{\partial^{2}\left(L^{(\bar{\sigma})} P\right)}{\partial \tau \partial x^{(\bar{e})}} \frac{\partial \psi}{\partial x^{(\bar{e})}} \mathrm{d} x \mathrm{~d} t \\
b_{T}(\psi)=\int_{0}^{T} \int_{\mathcal{B}} \frac{\partial F}{\partial \tau} \psi \mathrm{d} x \mathrm{~d} t+\int_{0}^{T} \sum_{\ell=1}^{N} \frac{\partial \Psi_{\ell}}{\partial \tau} \psi\left(O_{\ell}, t\right) \mathrm{d} t .
\end{gathered}
$$

According to $19, b_{T}$ is continuous on $L^{2}\left(0, T, H^{1}(\mathcal{B})\right), a_{T}$ is continuous and coercive on $L^{2}\left(0, T, H^{1}(\mathcal{B})\right)$, with a coercivity constant which is larger than $C \min \left\{1, T^{-2}\right\}$. Hence, the Lax-Milgram problem 2.14 admits a unique solution.

\subsection{Regularity of the solution}

\subsubsection{Regularity with respect to the space variable}

Using (2.14), we see that $\frac{\partial}{\partial t}\left(L^{(\bar{\sigma})} \frac{\partial P}{\partial x^{(\bar{e})}}\right) \in L^{2}\left(0, T, H_{d c}^{1}(\mathcal{B})\right)$. Integrating with respect to $t$ yields $L^{(\bar{\sigma})} \frac{\partial P}{\partial x} \in H_{00}^{1}\left(0, T, H_{d c}^{1}(\mathcal{B})\right)$.

As a consequence, since $L^{(\sigma)}: L^{2}(0, T) \rightarrow H_{00}^{1}(0, T)$ is invertible for $\sigma \in\left\{\sigma_{1}, \ldots, \sigma_{M}\right\}$, we have:

$$
\frac{\partial P}{\partial x^{(e)}} \in L^{2}\left(0, T, H_{d c}^{1}(\mathcal{B})\right) .
$$

Hence:

$$
P \in L^{2}\left(0, T, H_{d c}^{2}(\mathcal{B})\right)
$$

\subsubsection{Regularity with respect to the time variable}

Lemma 1: Let $k \geq 1$. Let us assume that $F \in H_{00}^{k+1}\left(0, T, L^{2}(\mathcal{B})\right)$ and that $\Psi_{\ell} \in H_{00}^{k+1}(0, T)$. Then:

$$
P \in H_{00}^{k}\left(0, T, H_{d c}^{2}(\mathcal{B})\right) \text {. }
$$

Proof:

Let $0 \leq s \leq k$ and $b_{T, s}$ be the linear form defined by:

$$
b_{T, s}(\psi)=\int_{0}^{T} \int_{\mathcal{B}} \frac{\partial^{s+1} F}{\partial \tau^{s+1}} \psi \mathrm{d} x \mathrm{~d} t+\int_{0}^{T} \sum_{\ell=1}^{N} \frac{\partial^{s+1} \Psi_{\ell}}{\partial \tau^{s+1}} \psi\left(O_{\ell}, t\right) \mathrm{d} t .
$$

Now let $P_{s}$ be the solution of $a_{T}\left(P_{s},.\right)=b_{T, s}($.$) . Then, using the uniqueness of the solution to$ Problem 2.14, we get that $P_{s}(., t)=\int_{0}^{t} P_{s+1}(., \tau) \mathrm{d} \tau$ whenever $0 \leq s<k$.

Indeed, for any $f \in L^{1}(0, T)$, letting $F(t)=\int_{0}^{t} f(\tau) \mathrm{d} \tau$, using integration by parts and $F(0)=0$, we get, for any $\sigma \in\left\{\sigma_{1}, \ldots, \sigma_{M}\right\}$ :

$$
L^{(\sigma)} F(t)=\int_{0}^{t} L^{(\sigma)} f(\tau) \mathrm{d} \tau
$$

Since $P_{k} \in L^{2}\left(0, T, H_{d c}^{2}(\mathcal{B})\right)$, we conclude that $P \in H_{00}^{k}\left(0, T, H_{d c}^{2}(\mathcal{B})\right)$.

\section{Galerkin method}

In this section, we reformulate the scheme 3.4 .2 as a Galerkin method in order to prove the convergence result. 


\subsection{Time discretization}

Let $k>0$ be a time step, such that $Q=\frac{T}{k}$ is an integer. Let us denote $t_{q}=q k$ for $q \in\{0,1, \ldots, Q\}$, $\mathbb{V}_{k}=\mathbb{P}_{k}^{0}\left(0, T, H^{1}(\mathcal{B})\right) \subset L^{2}\left(0, T, H^{1}(\mathcal{B})\right)$ where $\mathbb{P}_{k}^{0}(0, T)$ denotes the set of piecewise constant functions over the subdivision $\left(t_{0}=0, \ldots, t_{Q}=T\right)$. In the sequel the funtions $K^{(\sigma)}, L^{(\sigma)}\left(\phi^{q}\right), L^{(\sigma)}\left(\phi^{q^{\prime}}\right)$ are extended by zero on $\mathbb{R}_{-}^{*}$ for $\sigma \in\left\{\sigma_{1}, \ldots, \sigma_{M}\right\}$.

A numerical scheme on the graph is then defined as follows: find $\mathbf{p} \in \mathbb{V}_{k}$ such that:

$$
\forall \psi \in \mathbb{V}_{k}, \quad a_{T, k}(\mathbf{p}, \psi)=b_{T}(\psi)
$$

where $a_{T, k}$ is an approximation of $a_{T}$ to be specified below.

We use separation of space and time variables. Let us denote $\phi^{q}=\mathbf{1}_{\left[t_{q}, t_{q+1}[\text {. Then, for any }\right.}$ $u, v \in H^{1}(\mathcal{B}), q, q^{\prime} \in\{0 \ldots Q\}$, We get:

$$
\begin{aligned}
a_{T}\left(u \phi^{q^{\prime}}, v \phi^{q}\right) & =\int_{t_{q}}^{t_{q+1}} \int_{\mathcal{B}} \frac{\partial\left(L^{(\bar{\sigma})} \phi^{q^{\prime}}\right)}{\partial \tau} \frac{\partial u}{\partial x^{(\bar{e})}} \frac{\partial v}{\partial x^{(\bar{e})}} \mathrm{d} x \mathrm{~d} \tau \\
& =\int_{\mathcal{B}}\left(\left(L^{(\bar{\sigma})} \phi_{q^{\prime}}\right)\left(t_{q+1}\right)-\left(L^{(\bar{\sigma})} \phi_{q^{\prime}}\right)\left(t_{q}\right)\right) \frac{\partial u}{\partial x^{(\bar{e})}} \frac{\partial v}{\partial x^{(\bar{e})}} \mathrm{d} x \mathrm{~d} \tau \\
& =\int_{t_{q^{\prime}}}^{t_{q^{\prime}+1}} \int_{\mathcal{B}}\left(K^{(\bar{\sigma})}\left(t_{q+1}-\tau\right)-K^{(\bar{\sigma})}\left(t_{q}-\tau\right)\right) \frac{\partial u}{\partial x^{(\bar{e})}} \frac{\partial v}{\partial x^{(\bar{e})}} \mathrm{d} x \mathrm{~d} \tau
\end{aligned}
$$

Denoting for any $\sigma \in\left\{\sigma_{1}, \ldots, \sigma_{M}\right\}: K_{q}^{(\sigma)}=\frac{1}{k} \int_{t_{q}}^{t_{q+1}} K^{(\sigma)}(t) \mathrm{d} t$ for $q \geq 0$ and $K_{q}^{(\sigma)}=0$ for $q \leq 0$, then:

$$
a_{T}\left(u \phi^{q^{\prime}}, v \phi^{q}\right)=k \int_{\mathcal{B}}\left(K_{q-q^{\prime}}^{(\bar{\sigma})}-K_{q-q^{\prime}-1}^{(\bar{\sigma})}\right) \frac{\partial u}{\partial x^{(\bar{e})}} \frac{\partial v}{\partial x^{(\bar{e})}} \mathrm{d} x .
$$

Note that:

$$
\begin{aligned}
& \text { - } K_{q}^{(\sigma)}-K_{q-1}^{(\sigma)}=k \int_{-1}^{1}\left(K^{(\sigma)}\right)^{\prime}(k(q+t))(1-|t|) \mathrm{d} t \text { if } q>1, \\
& \text { - } K_{0}^{(\sigma)}-K_{-1}^{(\sigma)}=K_{0}^{(\sigma)}, \\
& \text { - } K_{q}^{(\sigma)}-K_{q-1}^{(\sigma)}=0 \text { if } q<-1 .
\end{aligned}
$$

Now, the approximation $a_{T, k}$ of $a_{T}$ is any bilinear form defined on $\mathbb{V}_{k}$ by:

$$
a_{T, k}\left(u \phi^{q}, v \phi^{q^{\prime}}\right)=k \int_{\mathcal{B}}\left(\tilde{K}_{q-q^{\prime}}^{(\bar{\sigma})}-\tilde{K}_{q-q^{\prime}-1}^{(\bar{\sigma})}\right) \frac{\partial u}{\partial x^{(\bar{e})}} \frac{\partial v}{\partial x^{(\bar{e})}} \mathrm{d} x
$$

such that the $\tilde{K}_{q}^{(\sigma)}$ are approximations of the $K_{q}^{(\sigma)}$ satisfying $\tilde{K}_{q}^{(\sigma)}=0$ for $q \leq 0$. Note in addition that:

$$
b_{T}\left(v \phi^{q}\right)=\int_{\mathcal{B}}\left(F\left(x, t_{q+1}\right)-F\left(x, t_{q}\right)\right) v(x) \mathrm{d} x+\sum_{\ell=1}^{N}\left(\Psi_{\ell}\left(t_{q+1}\right)-\Psi_{l}\left(t_{q}\right)\right) v\left(O_{\ell}\right) .
$$

The time discretized problem (3.17) has a unique solution if $a_{T, k}$ is continuous and coercive.

\subsection{Continuity and coercivity of the approximate bilinear form}

We give two different sufficient conditions for $a_{T, k}$ to be continuous and coercive: one which relies on the smallness of $\theta(k)$ defined in 1.12); and another one which relies on properties of $\left(\tilde{K}_{q}^{(\sigma)}\right)$ only. These conditions can be seen as stability conditions for the numerical scheme and will be used to prove Theorems 1 and 2 .

Lemma 2. Let $\left(A_{q}\right)_{0 \leq q \leq Q-1}$ be given real numbers, and $a$ be a bilinear form defined by:

$$
a:\left\{\begin{array}{l}
\mathbb{P}_{k}^{0}(0, T) \times \mathbb{P}_{k}^{0}(0, T) \rightarrow \mathbb{R} \\
(b, c)=\left(\sum_{q=0}^{Q-1} b_{q} \phi^{q}, \sum_{q=0}^{Q-1} c_{q} \phi^{q}\right) \mapsto k \sum_{q=0}^{Q-1} b_{q} \sum_{\tilde{q}=0}^{q} A_{\tilde{q}} c_{q-\tilde{q}}
\end{array}\right.
$$


Then: $|a(b, c)| \leq\|b\|_{L^{2}}\|c\|_{L^{2}} \sum_{q=0}^{Q-1}\left|A_{q}\right|$.

Proof: Let $a, b, c$ be as in the lemma. Using a change of variable and Cauchy-Schwarz inequality, we get:

$$
|a(b, c)|=\left|\sum_{\tilde{q}=0}^{Q-1} A_{\tilde{q}} k \sum_{q=\tilde{q}}^{Q-1} b_{q} c_{q-\tilde{q}}\right| \leq k \sum_{\tilde{q}=0}^{Q-1}\left|A_{\tilde{q}}\right|\left(\sum_{q=0}^{Q-1} b_{q}^{2}\right)^{1 / 2}\left(\sum_{q=0}^{Q-1} c_{q}^{2}\right)^{1 / 2}=\sum_{\tilde{q}=0}^{Q-1}\left|A_{\tilde{q}}\right|\|b\|_{L^{2}}\|c\|_{L^{2}} .
$$

Lemma 3. The bilinear form $a_{T, k}$ defined above is continuous and satisfies, for any $u, v$ in $\mathbb{P}^{0}\left(0, T, H^{1}(\mathcal{B})\right)$ :

$$
\left|a_{T, k}(u, v)\right| \leq \max _{1 \leq i \leq M} \sum_{q^{\prime}=0}^{Q-1}\left|\tilde{K}_{q^{\prime}}^{\left(\sigma_{i}\right)}-\tilde{K}_{q^{\prime}-1}^{\left(\sigma_{i}\right)}\right|\|u\|_{L^{2}\left(0, T, H^{1}(\mathcal{B})\right)}\|v\|_{L^{2}\left(0, T, H^{1}(\mathcal{B})\right)} .
$$

Proof:

$$
\begin{aligned}
& \text { Let } u=\sum_{q=0}^{Q-1} u_{q} \phi^{q}, v=\sum_{q=0}^{Q-1} v_{q} \phi^{q} \in \mathbb{P}^{0}\left(0, T, H^{1}(\mathcal{B})\right) \text {. Then, } \\
& \qquad \begin{aligned}
\left|a_{T, k}(u, v)\right| & \leq \sum_{i=1}^{M} k \sum_{q=0}^{Q-1} \sum_{q^{\prime}=0}^{q}\left|\tilde{K}_{q^{\prime}}^{\left(\sigma_{i}\right)}-\tilde{K}_{q^{\prime}-1}^{\left(\sigma_{i}\right)}\right| \int_{e_{i}}\left|\frac{\partial u_{q}}{\partial x^{\left(e_{i}\right)}} \frac{\partial v_{q-q^{\prime}}}{\partial x^{\left(e_{i}\right)}}\right| \mathrm{d} x \\
& \leq \sum_{i=1}^{M} k \sum_{q=0}^{Q-1} \sum_{q^{\prime}=0}^{q}\left|\tilde{K}_{q^{\prime}}^{\left(\sigma_{i}\right)}-\tilde{K}_{q^{\prime}-1}^{\left(\sigma_{i}\right)}\right||| u_{q}\left\|_{H^{1}\left(e_{i}\right)}\right\| v_{q-q^{\prime}} \|_{H^{1}\left(e_{i}\right)} .
\end{aligned}
\end{aligned}
$$

Using Lemma 2 and Cauchy-Schwarz inequality, we get:

$$
\begin{aligned}
\left|a_{T, k}(u, v)\right| & \leq \sum_{i=1}^{M} \sum_{q^{\prime}=0}^{Q-1}\left|\tilde{K}_{q^{\prime}}^{\left(\sigma_{i}\right)}-\tilde{K}_{q^{\prime}-1}^{\left(\sigma_{i}\right)}\right|\|u\|_{L^{2}\left(0, T, H^{1}\left(e_{i}\right)\right)}\|v\|_{L^{2}\left(0, T, H^{1}\left(e_{i}\right)\right)} \\
& \leq \max _{1 \leq i \leq M} \sum_{q^{\prime}=0}^{Q-1}\left|\tilde{K}_{q^{\prime}}^{\left(\sigma_{i}\right)}-\tilde{K}_{q^{\prime}-1}^{\left(\sigma_{i}\right)}\right| \sum_{i=1}^{M}\|u\|_{L^{2}\left(0, T, H^{1}\left(e_{i}\right)\right)}\|v\|_{L^{2}\left(0, T, H^{1}\left(e_{i}\right)\right)} \\
& \leq \max _{1 \leq i \leq M} \sum_{q^{\prime}=0}^{Q-1}\left|\tilde{K}_{q^{\prime}}^{\left(\sigma_{i}\right)}-\tilde{K}_{q^{\prime}-1}^{\left(\sigma_{i}\right)}\right|\|u\|_{L^{2}\left(0, T, H^{1}(\mathcal{B})\right)}\|v\|_{L^{2}\left(0, T, H^{1}(\mathcal{B})\right)}
\end{aligned}
$$

As a direct application of the lemma, we get the following estimate on the approximation of $a_{T}$.

Proposition 1. The following estimate holds :

$$
\left\|a_{T, k}-a_{T}\right\| \leq \theta(k)
$$

where the norm is the norm for bilinear forms on $L^{2}\left(0, T, H^{1}(\mathcal{B})\right)$. In addition, if $a_{T}$ is $\alpha$-coercive with $\alpha>\theta(k)$, then $a_{T, k}$ is $(\alpha-\theta(k))$-coercive, and continuous.

In Proposition 1, $\alpha$ depends on $T$. Hence, for a given $k, a_{T, k}$ may not be coercive for large $T$. The following lemma gives a condition under which $a_{T, k}$ is coercive for any $k$ and $T$. It is a refinement of the Lemma 4.2 of [14].

Lemma 4. Let $k, C, E, T_{m} \in \mathbb{R}^{+*}$ and $\left(K_{q}\right)_{q \in \mathbb{Z}}$ such that:

- $k<\min \left\{T_{m}, T\right\}$,

- $0 \leq K_{q} \leq C$ if $q \geq 0$,

- $K_{q}=0$ if $q<0$, 
- $K_{q+1}-2 K_{q}+K_{q-1} \geq 0$ if $q \geq 1$,

- $E \leq \frac{K_{q+1}-2 K_{q}+K_{q-1}}{k^{2}}$ if $T_{m} \leq q k \leq 2 T_{m}$.

Let $a$ be the bilinear form defined as in Lemma 2 with $A_{q}=K_{q}-K_{q-1}$. Then

$$
\left\{\begin{array}{l}
|a(b, c)| \leq C\|b\|_{L^{2}}\|c\|_{L^{2}}, \\
a(b, b) \geq \alpha_{T}\|b\|_{L^{2}}^{2},
\end{array}\right.
$$

where:

$$
\alpha_{T}=\frac{1}{2} T_{m}^{2} E \min \left\{\frac{T_{m}^{2}}{16 T^{2}}, \frac{1}{40}\right\} .
$$

Remark: The assumptions on $\left(K_{q}\right)$ are discrete analogues of the fact that the $\left(K^{(\sigma)}\right)$ are nonnegative and bounded, $\left(K^{(\sigma)}\right)^{\prime \prime}$ are nonnegative and the $\left(K^{(\sigma)}\right)^{\prime \prime}$ is larger than some constant $E>0$ on an interval $\left[T_{m}, 2 T_{m}\right]$, for any $\sigma \in\left\{\sigma_{1}, \ldots, \sigma_{M}\right\}$. This fact arises straightforwardly from the generalized Dirichlet series expression in Lemma 2 in [10] (see also the forthcoming second part of this article).

Proof: $\left(K_{q+1}-K_{q}\right)_{q \geq 0}$ is increasing and bounded by $2 C$ and therefore convergent. Its limit is zero, otherwise $\left(K_{q}\right)$ would not be bounded. Thus, $\left(K_{q+1}-K_{q}\right)_{q \geq 0}$ is negative and so $\left(K_{q}\right)_{q \geq 0}$ is decreasing. We have, from Proposition 1 that

$$
\begin{aligned}
|a(b, c)| & \leq\|b\|_{L^{2}}\|c\|_{L^{2}}\left(K_{0}+\sum_{q=1}^{Q-1}\left|K_{q}-K_{q-1}\right|\right) \\
& \leq\|b\|_{L^{2}}\|c\|_{L^{2}}\left(K_{0}+K_{0}-K_{Q-1}\right) \leq 2 C\|b\|_{L^{2}}\|c\|_{L^{2}} .
\end{aligned}
$$

Let $b=\sum_{q=0}^{Q-1} b_{q} \phi^{q}, c=\sum_{q=0}^{Q-1} c_{q} \phi^{q}$. Let $\hat{b}, \hat{c}, G$ be the discrete Fourier transform of $\left(k b_{q}\right)_{q},\left(k c_{q}\right)_{q}$, $\left(K_{q}-K_{q-1}\right)_{q}$ :

$$
\hat{b}(\xi)=k \sum_{q=0}^{Q-1} b_{q} e^{-2 \pi i q k \xi}, \hat{c}(\xi)=k \sum_{q=0}^{Q-1} c_{q} e^{-2 \pi i q k \xi}, G(\xi)=\sum_{q \in \mathbb{Z}}\left(K_{q}-K_{q-1}\right) e^{-2 \pi i q k \xi} .
$$

Then, according to Parseval equality for Fourier series, we have:

$$
\int_{0}^{T} b(t)^{2} \mathrm{~d} t=k \sum_{q=0}^{Q-1} b_{q}^{2}=\int_{-\frac{1}{2 k}}^{\frac{1}{2 k}}|\hat{b}(\xi)|^{2} \mathrm{~d} \xi, \quad a(b, c)=\int_{-\frac{1}{2 k}}^{\frac{1}{2 k}} G(\xi) \hat{b}(\xi) \hat{c}(\xi) \mathrm{d} \xi .
$$

Let $D_{n}(x)=1+2 \sum_{q=1}^{n} \cos (q x)$ be the Dirichlet kernel. By summing by parts, and using the decrease of $\left(K_{q}\right)_{q \geq 0}$, we obtain:

$$
\begin{aligned}
\operatorname{Re} G(\xi) & =\sum_{q=1}^{\infty}\left(K_{q}-K_{q-1}\right)(\cos (2 \pi q k \xi)-1)+\lim _{q \rightarrow \infty}\left(K_{0}-K_{q}\right) \\
& \geq\left(K_{1}-K_{0}\right)(1-1)+\sum_{q=1}^{\infty}\left(K_{q+1}-2 K_{q}+K_{q-1}\right) \sum_{\tilde{q}=0}^{q}(1-\cos (2 \pi \tilde{q} k \xi)) \\
& \geq \sum_{q=1}^{\infty}\left(K_{q+1}-2 K_{q}+K_{q-1}\right)\left(q+\frac{1}{2}-\frac{1}{2} D_{q}(2 \pi k \xi)\right)
\end{aligned}
$$

- For $x \in]-\frac{\pi}{4 n}, \frac{\pi}{4 n}\left[\backslash\{0\}, D_{n}^{\prime \prime}(x) \leq-\sum_{q=1}^{n} q^{2} \cos \frac{\pi}{4}=-\frac{\sqrt{2}}{2} \frac{n(n+1)(2 n+1)}{6} \leq-\frac{n^{3}}{6}\right.$. Вy integrating twice, we get that $D_{n}(x) \leq 2 n+1-\frac{n^{3}}{12} x^{2}$. 
- For $x \in]-\pi, \pi[\backslash] \frac{-\pi}{2 n}, \frac{\pi}{2 n}\left[\right.$, we have $\left|\sin \frac{x}{2}\right| \geq\left|\sin \frac{\pi}{4 n}\right|=\frac{\pi}{4 n}\left|\operatorname{sinc} \frac{\pi}{4 n}\right| \geq \frac{\pi}{4 n}\left|\operatorname{sinc} \frac{\pi}{4}\right| \geq \frac{2}{3 n}$, and then $\left|D_{n}(x)\right|=\left|\frac{\sin \left(n+\frac{1}{2}\right) x}{\sin \frac{x}{2}}\right| \leq \frac{1}{\left|\sin \frac{x}{2}\right|} \leq \frac{3 n}{2}$ (sin, sinc are monotone on $\left[0, \frac{\pi}{4}\right]$, where sinc is defined by: $\left.\operatorname{sinc}(x)=\frac{\sin x}{x}\right)$.

- For $x \in]-\frac{\pi}{2 n}, \frac{\pi}{2 n}[\backslash] \frac{-\pi}{4 n}, \frac{\pi}{4 n}\left[, D_{n}^{\prime}(x)\right.$ has the same sign as $-x$. Hence

$$
\begin{gathered}
D_{n}(x) \leq 2 n+1-\frac{n^{3}}{12}\left(\frac{\pi}{4 n}\right)^{2} \leq 2 n+1-\frac{n}{20}, \\
\left.q+\frac{1}{2}-\frac{1}{2} D_{q}(2 \pi k \xi) \geq \min \left\{\frac{1}{24} q^{3}(2 \pi k \xi)^{2}, \frac{q}{40}, q+\frac{1}{2}-\frac{3 q}{4}\right\} \text { for } \xi \in\right] \frac{-1}{2 k}, \frac{1}{2 k}[.
\end{gathered}
$$

Then

$$
\begin{gathered}
\operatorname{Re} G(\xi) \geq \sum_{T_{m} \leq q k \leq 2 T_{m}}\left(K_{q+1}-2 K_{q}+K_{q-1}\right) \min \left\{\frac{1}{24} q^{3}(2 \pi k \xi)^{2}, \frac{q}{40}\right\}, \\
\operatorname{Re} G(\xi) \geq \frac{T_{m}}{k} k^{2} E \min \left\{\frac{1}{24}\left(\frac{T_{m}}{k}\right)^{3}(2 \pi k \xi)^{2}, \frac{1}{40} \frac{T_{m}}{k}\right\}, \\
\operatorname{Re} G(\xi) \geq T_{m}^{2} E \min \left\{T_{m}^{2} \xi^{2}, \frac{1}{40}\right\} .
\end{gathered}
$$

Using Cauchy-Schwarz inequality, we also have:

$$
\|\hat{b}\|_{\infty} \leq \sqrt{T}\|b\|_{L^{2}}
$$

Hence, $\int_{-\frac{1}{4 T}}^{\frac{1}{4 T}}|\hat{b}(\xi)|^{2} \mathrm{~d} \xi \leq \frac{1}{2 T}\|\hat{b}\|_{\infty}^{2} \leq \frac{1}{2}\|b\|_{L^{2}}^{2}$ and $\frac{1}{2}\|b\|_{L^{2}}^{2} \leq \int_{]-\frac{1}{2 k}, \frac{1}{2 k}[\backslash] \frac{1}{4 T},-\frac{1}{4 T}[}|\hat{b}(\xi)|^{2} \mathrm{~d} \xi$, and therefore:

$$
a(b, b) \geq \int_{]-\frac{1}{2 k}, \frac{1}{2 k}[\backslash]-\frac{1}{4 T}, \frac{1}{4 T}[} \operatorname{Re} G(\xi)|\hat{b}(\xi)|^{2} \mathrm{~d} \xi \geq \int_{]-\frac{1}{2 k}, \frac{1}{2 k}[\backslash]-\frac{1}{4 T}, \frac{1}{4 T}[} 2 \alpha_{T}|\hat{b}(\xi)|^{2} \mathrm{~d} \xi \geq \alpha_{T}\|b\|_{L^{2}}^{2} .
$$

Proposition 2. Assume that $\left(\tilde{K}_{q}^{(\sigma)}\right)_{q \in \mathbb{Z}}$ satisfies the hypotheses of Lemma 4 for each $\sigma \in\left\{\sigma_{1}, \ldots, \sigma_{M}\right\}$. Let $D=\sum_{i=1}^{M}\left|e_{i}\right|$. Then $a_{T, k}$, as defined in 3.18 , is continuous and coercive:

$$
\begin{gathered}
\forall p, c \in L^{2}\left(0, T, H^{1}(\mathcal{B})\right), \quad\left|a_{T, k}(p, c)\right| \leq 2 C\|p\|_{L^{2}\left(0, T, H^{1}(\mathcal{B})\right)}\|c\|_{L^{2}\left(0, T, H^{1}(\mathcal{B})\right)}, \\
\forall p \in L^{2}\left(0, T, H^{1}(\mathcal{B})\right), \quad a_{T, k}(p, p) \geq \frac{\alpha_{T}}{\sqrt{1+D^{2}}}\|p\|_{L^{2}\left(0, T, H^{1}(\mathcal{B})\right)}^{2} .
\end{gathered}
$$

\section{Proof:}

Continuity is a direct consequence of Proposition 1 and Lemma 4.

For $c \in H^{1}(\mathcal{B}),\left|c(M)-c\left(O_{1}\right)\right| \leq \int_{\mathcal{B}}\left|\frac{\partial c}{\partial x^{(\bar{e})}}\right| \mathrm{d} x \leq \sqrt{\int_{\mathcal{B}} \mathrm{d} x \int_{\mathcal{B}}\left|\frac{\partial c}{\partial x^{(\bar{e})}}\right|^{2} \mathrm{~d} x}$.

It follows that the Poincaré inequality: $\|p\|_{H^{1}(\mathcal{B})}^{2} \leq\left(D^{2}+1\right) \int_{\mathcal{B}}\left|\frac{\partial p}{\partial x^{(\bar{e})}}\right|^{2} \mathrm{~d} x$ holds true.

Then, using Lemma 4 with $a=a_{T, k}$, for $p \in L^{2}\left(0, T, H^{1}(\mathcal{B})\right)$, we have that

$$
\begin{array}{r}
a_{T, k}(p, p) \geq \alpha_{T} \int_{\mathcal{B}} \int_{0}^{T}\left|\frac{\partial p}{\partial x^{(\bar{e})}}\right|^{2} \mathrm{~d} x \mathrm{~d} t \\
\geq \alpha_{T} \int_{0}^{T} \frac{1}{\sqrt{D^{2}+1}}\|p(., t)\|_{H^{1}(\mathcal{B})}^{2} \mathrm{~d} t \\
\geq \frac{\alpha_{T}}{\sqrt{D^{2}+1}}\|p\|_{L^{2}\left(0, T, H^{1}(\mathcal{B})\right)}^{2} .
\end{array}
$$




\subsection{Total discretization on the graph}

Let $h$ be a space step, $h>0$.

For each edge $e$, let us denote $h^{(e)}$ the largest space step such that $h^{(e)} \leq h$ and $S^{(e)}=\frac{|e|}{h^{(e)}}$ is an integer.

Let $0=x_{0}^{(e)}, x_{1}^{(e)}=h^{(e)} \ldots, x_{S^{(e)}}^{(e)}=S^{(e)} h^{(e)}=|e|$ be the subdivision $[0,|e|]$ with step $h^{(e)}$. For $s \in\left\{0, \ldots, S^{(e)}\right\}$, let $X_{s}^{(e)}$ designates the point of $e$ with last local coordinate equal to $x_{s}^{(e)}$ (so that if the local coordinate system has $O_{i}$ as origin then $\left.X_{s}^{(e)}=O_{i}+R^{(e)}\left(\hat{0}, x_{s}^{(e)}\right)\right)$.

Let $\mathbb{P}_{h}^{1}(\mathcal{B})$ be the subset of continuous functions $\psi: \mathcal{B} \rightarrow \mathbb{R}$ such that $\psi$ is affine on $\left[X_{r}^{(e)}, X_{r+1}^{(e)}\right]$ for each $r \in\left\{0, \ldots, S^{(e)}-1\right\}$ (i.e., in local coordinates, $\psi\left(O_{i}+R^{(e)}\left(\hat{0}, x^{(e)}\right)\right)$ is affine on $\left.\left[x_{s}^{(e)}, x_{s+1}^{(e)}\right]\right)$. Assume that $a_{T, k}$ is continuous coercive. Let us define $\mathbf{p}_{h, k} \in \mathbb{P}_{k}^{0}\left(0, T, \mathbb{P}_{h}^{1}(\mathcal{B})\right)$ by:

$$
\forall \psi \in \mathbb{P}_{k}^{0}\left(0, T, \mathbb{P}_{h}^{1}(\mathcal{B})\right), \quad a_{T, k}\left(\mathbf{p}_{h, k}, \psi\right)=b_{T, h}(\psi)
$$

where $b_{T, h}$ is an approximation of $b_{T}$ defined below.

Let $\mathcal{P}_{h}$ be the set of all $X_{s}^{(e)}$, with $e$ an edge of the graph and $s \in\left\{0, \ldots, S^{(e)}\right\}$, with $O_{1}$ excluded. For $X \in \mathcal{P}_{h}$, let us define $\psi^{X} \in \mathbb{P}_{h}^{1}(\mathcal{B})$ by $\psi^{X}(X)=1$ and $\psi^{X}(Y)=0$ if $Y \in \mathcal{P}_{h}$ and $Y \neq X$.

We approximate the integral on $\mathcal{B}$ in $b_{T}$ by the trapezoidal rule. So, we define $b_{T, h}$ by:

$$
b_{T, h}\left(\psi^{X} \phi^{q}\right)=\left(F\left(X, t_{q+1}\right)-F\left(X, t_{q}\right)\right) \int_{\mathcal{B}} \psi^{X}(x) \mathrm{d} x+\sum_{\ell=1}^{N} \psi^{X}\left(O_{\ell}\right)\left(\Psi_{\ell}\left(O_{\ell}, t_{q+1}\right)-\Psi_{\ell}\left(O_{\ell}, t_{q}\right)\right),
$$

for every $X \in \mathcal{P}_{h}$ and $q \in\{0, \ldots, Q-1\}$. Note that if $F=0$, then $b_{T, h}=b_{T}$, and that for any $\psi \in \mathbb{P}_{h}^{1}(\mathcal{B})$ and $f \in H_{d c}^{r}(\mathcal{B}), r \in\{1,2\}$, we have:

$$
\left|\int_{\mathcal{B}} f \psi \mathrm{d} x-\sum_{X \in \mathcal{P}_{h}} f(X) \psi(X) \int_{\mathcal{B}} \psi^{X} \mathrm{~d} x\right| \leq h^{r} \int_{\mathcal{B}}\left|\frac{\partial^{r}(f \psi)}{\left(\partial x^{(\bar{e})}\right)^{r}}\right| \mathrm{d} x .
$$

In the right handside, $\int_{\mathcal{B}}$ is to be understood as a sum of integrals on each step of the space subdivision: over these intervals, $\psi$ is smooth and the $r^{t h}$-derivative of $f \psi$ is in $L^{1}$. Furthermore:

$$
\begin{gathered}
\int_{\mathcal{B}}\left|\frac{\partial(f \psi)}{\partial x^{(\bar{e})}}\right| \mathrm{d} x \leq h\left(\|f\|_{H_{d c}^{1}(\mathcal{B})}\|\psi\|_{L^{2}(\mathcal{B})}+\|f\|_{L^{2}(\mathcal{B})}\|\psi\|_{H_{d c}^{1}(\mathcal{B})}\right) . \\
\int_{\mathcal{B}}\left|\frac{\partial^{2}(f \psi)}{\left(\partial x^{(\bar{e})}\right)^{2}}\right| \mathrm{d} x \leq h^{2}\left(\|f\|_{H_{d c}^{2}(\mathcal{B})}\|\psi\|_{L^{2}(\mathcal{B})}+2\|f\|_{H_{d c}^{1}(\mathcal{B})}\|\psi\|_{H_{d c}^{1}(\mathcal{B})}+0\right) \text { if } r=2 .
\end{gathered}
$$

Hence, if $F \in H^{1}\left(0, T, H_{d c}^{r}(\mathcal{B})\right)$, then, by using Cauchy-Schwarz inequality:

$$
\left|\left(b_{T, h}-b_{T}\right)\left(\psi \phi^{q}\right)\right| \leq 3 h^{r} \sqrt{k}\|F\|_{H^{1}\left(\left[t_{q}, t_{q+1}\right], H_{d c}^{r}(\mathcal{B})\right)}\|\psi\|_{H^{1}(\mathcal{B})}
$$

and therefore, for $\psi \in \mathbb{P}_{k}^{0}\left(0, T, \mathbb{P}_{h}^{1}(\mathcal{B})\right)$ and $F \in H^{1}\left(0, T, H_{d c}^{r}(\mathcal{B})\right), r \in\{1,2\}$, we have:

$$
\left|\left(b_{T, h}-b_{T}\right)(\psi)\right| \leq 3 h^{r}\|F\|_{H^{1}\left(0, T, H_{d c}^{r}(\mathcal{B})\right)}\|\psi\|_{L^{2}\left(0, T, H^{1}(\mathcal{B})\right)}
$$

Now, rewriting

$$
\mathbf{p}_{h, k}=\sum_{X \in \mathcal{P}_{h}, 0 \leq q \leq Q-1} P_{X, q} \psi^{X} \phi^{q}
$$

and introducing the notations

$$
\begin{gathered}
K_{q, X, Y}=\int_{\mathcal{B}} \tilde{K}_{q}^{(\bar{\sigma})} \frac{\partial \psi^{X}}{\partial x^{(\bar{e})}} \frac{\partial \psi^{Y}}{\partial x^{(\bar{e})}} \mathrm{d} x \\
R_{X, q}=b_{T, h}\left(\psi^{X} \sum_{\tilde{q}=0}^{q-1} \phi^{\tilde{q}}\right)=b_{T, h}\left(\psi^{X} \mathbf{1}_{\left[t_{0}, t_{q}\right]}\right),
\end{gathered}
$$


the totally discretized problem (3.19) is equivalent to solve for the coordinates $P_{q, X}$ of $\mathbf{p}_{h, k}$ the following system:

$\forall r \in\{0, \ldots, Q-1\}, X \in \mathcal{P}_{h}: \sum_{Y \in \mathcal{P}_{h}}\left(K_{0, X, Y} P_{Y, r}+\sum_{\tilde{q}=0}^{r-1}\left(K_{r-\tilde{q}, X, Y}-K_{r-\tilde{q}-1, X, Y}\right) P_{Y, \tilde{q}}\right)=R_{X, r+1}-R_{X, r}$.

Since $R_{0, X}=0$, by summation over $r$ this can be reformulated as:

$$
\forall q \in\{0, \ldots, Q-1\}, X \in \mathcal{P}_{h}: \sum_{\tilde{q}=0}^{q} \sum_{Y \in \mathcal{P}_{h}} K_{q-\tilde{q}, X, Y} P_{Y, \tilde{q}}=R_{X, q+1} .
$$

Note that

$$
R_{X, q}= \begin{cases}\Psi_{\ell}\left(t_{q}\right)+\frac{1}{2} \sum_{1 \leq j \leq M, O_{\ell} \in e_{j}} h^{\left(e_{j}\right)} F\left(O_{\ell}, t_{q}\right) & \text { if } X=O_{\ell}, \\ h^{(e)} F\left(X_{s}^{(e)}, t_{q+1}\right) & \text { if } X=X_{s}^{(e)}, s \in\left\{1, \ldots, S^{(e)}-1\right\},\end{cases}
$$

and

$$
K_{q, X, Y}= \begin{cases}\sum_{1 \leq j \leq M, O_{\ell} \in e_{j}} \frac{\tilde{K}_{q}^{\left(\sigma_{j}\right)}}{h^{\left(e_{j}\right)}} & \text { if } X=Y=O_{\ell}, \\ \frac{2 \tilde{K}_{q}^{\left(\sigma_{j}\right)}}{h^{\left(e_{j}\right)}} & \text { if } X=Y=X_{s}^{\left(e_{j}\right)}, s \in\left\{1, \ldots, S^{\left(e_{j}\right)}-1\right\}, \\ \frac{-\tilde{K}_{q}^{\left(\sigma_{j}\right)}}{h^{\left(e_{j}\right)}} & \text { if }\{X, Y\}=\left\{X_{s}^{\left(e_{j}\right)}, X_{s+1}^{\left(e_{j}\right)}\right\}, s \in\left\{0, \ldots, S^{\left(e_{j}\right)}-1\right\}, \\ 0 & \text { otherwise. }\end{cases}
$$

Hence, we have the same scheme as described in the introduction, Section 1.2 .

\subsection{Discretization in the cross-section}

If we want to use Theorem 1 and 2 of Section 4 we must determine an approximation of $K_{q}^{(\sigma)}$ such that $\theta(k)$ is small.

In Section 5, we test the scheme on the graph with the exact value of the kernel when known, and with the three different approximations for the kernel, described below: 33.26$),(3.29),(3.30)$.

\subsubsection{Exact kernels}

When the cross-section $\sigma$ is a rectangle, an equilateral triangle or a disc, we express in $\left[8 K^{(\sigma)}\right.$ as a computable generalized Dirichlet series:

$$
\begin{aligned}
& \left.K^{(\sigma)}(t)=\sum_{k=-\infty}^{\infty} \frac{4}{(2 k+1)^{2} \pi^{2}} e^{-\pi^{2}(2 k+1)^{2} t} \quad \text { if } \sigma=\right] 0,1[, \\
& \left.K^{(\sigma)}(t)=a b K^{(] 0,1[)}\left(a^{-2} t\right) K^{(] 0,1[)}\left(b^{-2} t\right) \quad \text { if } \sigma=\right] 0, a[\times] 0, b[\text {, } \\
& K^{(\sigma)}(t)=\quad 4 \pi \sum_{i=1}^{+\infty} \frac{1}{\mu_{i}{ }^{2}} e^{-\mu_{i}{ }^{2} t} \quad \text { if } \sigma=\left\{x \in \mathbb{R}^{2} ;\|x\|_{2}<1\right\}, \\
& K^{(\sigma)}(t)=\frac{3 \sqrt{3}}{2 \pi^{2}} \sum_{k=1}^{+\infty} \frac{1}{k^{2}} \exp \left(-\frac{16 \pi^{2}}{3} k^{2} t\right) \quad \text { if } \sigma=\mathcal{T}
\end{aligned}
$$

where $\mu_{i}$ denotes the $i$-th zero of the zero-th Bessel function $J_{0}$ and $\mathcal{T}$ denotes the triangle with vertices $(0,0),(0,1),\left(\frac{1}{2}, \frac{\sqrt{3}}{2}\right)$. 
If these exact expressions are used, then $\theta(k)=0$. For the square and the triangle cross section, we obtain series expansions for the kernels that converge rapidly for small times, by using the Poisson summation formula.

Furthermore, one can show that $\left(K_{q}^{(\sigma)}\right)$ satisfies the hypotheses of Proposition 2. Hence, with this choice, the scheme on the graph is unconditionally stable.

\subsubsection{Numerical approximation of the kernels}

For an arbitrary smooth domain $\sigma$, let us consider a quasi-uniform triangulation of $\sigma$ and denote by $H$ the diameter of its largest triangle. Let $S_{H}^{(\sigma)} \subset L^{2}(\sigma)$ be the space of $\mathbb{P}^{r-1}$ finite elements where $r \geq 2$. Let $T_{H}^{(\sigma)}: L^{2}(\sigma) \rightarrow S_{H}^{(\sigma)}$ be the operator discretizing the inverse of the Dirichlet Laplacian, using Nitsche method for the boundary conditions. Then, $\left(T_{H}^{(\sigma)}-\tau I\right): S_{H}^{(\sigma)} \rightarrow S_{H}^{(\sigma)}$ is invertible whenever $\tau \geq 0$.

Consider the following numerical scheme which use the implicit Euler method as time integrator:

$$
\left\{\begin{array}{l}
V_{H, k}^{0}=W^{(\sigma)} \\
T_{H}^{(\sigma)} \frac{V_{H, k}^{q+1}-V_{H, k}^{q}}{k}=V_{H, k}^{q+1} \\
K_{q}^{(\sigma)}=\int_{\sigma}^{k} V_{H, k}^{q} \mathrm{~d} \hat{x},
\end{array}\right.
$$

where $W^{(\sigma)}$ is an approximation of 1 . Note that there is a special method of resolution which is presented in Section 3.5.3.

In the present paper, we test the case where $W^{(\sigma)}$ is the interpolant of $(I-k \Delta)^{-1} 1$ and can be computed explicitly.

If $W=1$, then we show in [8] that $\left(\tilde{K}_{q}^{(\sigma)}\right)$ satisfies the hypotheses of Proposition 2 . Hence, with this choice, the scheme on the graph is unconditionally stable. Using techniques in Thomée's book 30 . we also prove that when $h=k^{\gamma}, \gamma>0$, and $k$ small enough, the scheme is convergent with:

$$
\theta(k) \leq C \max \left\{H^{2}, k\right\}^{\frac{1}{3}} .
$$

The numerical experiments in [8] indicate that the power $1 / 3$ may be improved and replaced by $1 / 2$ and the use of $L$-stable higher-order methods do not improve the accuracy, unless an approximation for small times is used as in the next paragraph.

\subsubsection{Corrected approximation}

When the numerical approximation is used, most of the error is done for small time. Hence, if an asymptotic expansion near $t=0^{+}$is known, one can use it to obtain a more accurate approximation of the kernel.

When $\sigma$ is smooth and simply connected, using the boundary layer theory presented in Gie, Jung \& Temam [12, we obtain in [8] an asymptotic expansion of $K$.

Let us denote $\gamma:[0,|\partial \sigma|] \rightarrow \partial \sigma$, a parametrization by the arclength of $\partial \sigma$ (i.e. $\left\|\gamma^{\prime}\right\|=1$ ), $\kappa(s)=\operatorname{det}\left(\gamma^{\prime}(s), \gamma^{\prime \prime}(s)\right)$ the curvature. Then:

$$
K^{(\sigma)}(t)=|\sigma|-\frac{2}{\sqrt{\pi}}|\partial \sigma| t^{\frac{1}{2}}+\pi t+\frac{1}{6 \sqrt{\pi}} t^{\frac{3}{2}} \int_{0}^{|\partial \sigma|} \kappa(s)^{2} \mathrm{~d} s+O_{t \rightarrow 0^{+}}\left(t^{2}\right) .
$$

Let us consider a modified version of scheme (3.26), and take the BDF2 time integrator (see [30]) and $\mathbb{P}^{1}$-elements in space.

$$
\left\{\begin{array}{l}
V_{H, k}^{0}=1, \\
T_{H}^{(\sigma)} \frac{V_{H, k}^{1}-V_{H, k}^{0}}{k}=V_{H, k}^{1}, \\
T_{H}^{(\sigma)} \frac{3 V_{H, k}^{q+2}-4 V_{H, k}^{q+1}+V_{H, k}^{q}}{3 k}=V_{H, k}^{q+2} \quad \text { if } q \geq 0 \\
\tilde{K}_{q, \text { num }}^{(\sigma)}=\int_{\sigma} \frac{V_{H, k}^{q}+V_{H, k}^{q+1}}{2} \mathrm{~d} x .
\end{array}\right.
$$


Let us use the asymptotic expansion $(3.28)$ to correct the previous approximation for small times. From (3.28), $\int_{0}^{\tau} K^{(\sigma)} \mathrm{d} t=\chi(t)+O_{t \rightarrow 0^{+}}\left(t^{\frac{5}{2}}\right)$, with $\chi(t)=|\sigma| t-\frac{4}{3 \sqrt{\pi}}|\partial \sigma| t^{\frac{3}{2}}+\frac{\pi}{2} t^{2}$. We take $\tilde{K}_{q, \text { cor }}^{(\sigma)}$ to be such that:

$$
\begin{cases}K_{q, \text { cor }}^{(\sigma)}=K_{q, \text { num }}^{(\sigma)} & \text { if } Q^{\frac{1}{3}} \leq q \leq Q-1, \\ \frac{K_{q, \text { cor }}^{(\sigma)}-K_{q-1, \text { cor }}^{(\sigma)}}{k}=\frac{\chi((q+1) k)-2 \chi(q k)+\chi((q-1) k)}{k^{2}} & \text { if } 1 \leq q \leq Q^{\frac{1}{3}}\end{cases}
$$

In this case, the hypotheses of Proposition 2 are not automatically satisfied, but we can refine (3.27) and get, when $H=k^{\gamma}, \gamma \geq \frac{1}{2}$ and $k$ small enough:

$$
\theta(k) \leq C \max \{k, H\}^{\frac{2}{3}} .
$$

Numerical experiments suggest that the power $2 / 3$ may be replaced by 1 , or more if all the terms from $(3.28)$ are used.

\subsection{Remarks on the solving of the scheme}

Let us describe three methods to solve the scheme (3.21) on the graph. The first two methods can be used for any type of kernel approximation but the last one will only work for 3.26 .

Let $\mathbf{S}$ be the number of elements of $\mathcal{P}_{h}$.

\subsubsection{Using the block triangular structure}

The first computational strategy uses the block-triangular structure of the matrix with respect to the time variable and solves the corresponding system of equations by the method of forward substitution. In this case, only the sparse matrix $\left(K_{0, X, Y}\right)_{X, Y \in \mathcal{P}_{h}}$ needs to be inverted. It is tridiagonal, except for the junctions. Hence, its Choleski factorisation takes $O\left(N \mathbf{S}+N^{3}\right)$ flops. The Choleski factor has $O\left(N \mathbf{S}+N^{2}\right)$ nonzero elements. The computation of $\left(P_{X, q}\right)_{X \in \mathcal{P}_{h}}$ at the time step number $q$, when values $\left(P_{X, 0}\right)_{X \in \mathcal{P}_{h}}, \ldots,\left(P_{X, q-1}\right)_{X \in \mathcal{P}_{h}}$ are known, costs $O\left(q\left(N \mathbf{S}+N^{2}\right)\right)$ flops.

Hence, the total computational cost is $O\left(N \mathbf{S}+N^{3}+Q^{2}\left(N \mathbf{S}+N^{2}\right)\right)$.

\subsubsection{Using the Fast Fourier Transform}

Let us describe the second strategy. Denote $\left(A_{q}\right)_{0 \leq q \leq Q-1} \star\left(B_{q}\right)_{0 \leq q \leq Q-1}=\left(\sum_{\tilde{q}=0}^{q} A_{q} B_{q-\tilde{q}}\right)_{0 \leq q \leq Q-1}$.

This discrete convolution can be computed by using the Discrete Fourier Transform with only $O(Q \log (Q))$ multiplications. First, let us solve the equation:

$$
(K \star Z)_{q, X, Y}= \begin{cases}\delta_{X, Y} & \text { if } q=0, \\ 0 & \text { if } q \neq 0 .\end{cases}
$$

The solution $Z$ can be computed by $\left\lceil\log _{2} Q\right\rceil$ Newton's iterations:

$$
Z=\Phi^{\left\lceil\log _{2} Q\right\rceil}(A), A_{q}=\left\{\begin{array}{ll}
\left(\left(K_{0, X, Y}\right)_{X, Y \in \mathcal{P}_{h}}\right)^{-1} & \text { if } q=0 \\
0 & \text { if } q \neq 0
\end{array}, \quad \Phi(B)=B \star(2 I-B \star K \star B) .\right.
$$

Then $P_{X, q}$ is given by:

$$
\forall q \in\{0, \ldots, Q-1\}, X \in \mathcal{P}_{h}: P_{X, q}=\sum_{Y \in \mathcal{P}_{h}} \sum_{\tilde{q}=0}^{q} Z_{q, X, Y} R_{q+1, Y}
$$

The computational cost is here $O\left(Q \log (Q)^{2} \mathbf{S}^{3}\right)$.

Remark: When there is no forcing along the pipe $(F=0)$, the second method is more efficient for large $Q$ since one can take one space step per pipe without loss of precision, which implies $\mathbf{S}=N$. 


\subsubsection{Coupling with the cross-section}

The following formulation of the scheme is of interest when we want to recover the velocity inside the tubes. Besides, contrary to the two previous methods, its complexity scales linearly with the number of time steps.

For $x \in \mathcal{B}, \hat{x} \in \bar{\sigma}(x)$, let $U(x, \hat{x}, t)$ such that:

$$
\begin{cases}\frac{\partial U}{\partial t}(x, \hat{x}, t)-\Delta_{\hat{x}} U(x, \hat{x}, t)=-\frac{\partial P}{\partial x^{\bar{e}}}(x), & \hat{x} \in \sigma, t>0, \\ U(\hat{x}, t)=0, & \hat{x} \in \partial \sigma, t>0, \\ U(\hat{x}, 0)=0, & \hat{x} \in \sigma .\end{cases}
$$

Then [20, 21], at some distance from the junctions:

$$
u^{\varepsilon}\left(O_{i_{j}}+R^{\left(e_{j}\right)}\left(\varepsilon \hat{x}, x_{d}\right), t \varepsilon^{2}\right) \simeq R^{\left(e_{j}\right)}\left(\hat{0}, U\left(\hat{x}, O_{i_{j}}+R^{\left(e_{j}\right)}\left(\hat{0}, x_{d}\right), t\right) .\right.
$$

In the sequel, $U_{s+\frac{1}{2}, q}^{\left(e_{j}\right)}$ designates an approximation of $U\left(\cdot, X_{s+\frac{1}{2}}^{\left(e_{j}\right)}, t_{q+1 / 2}\right)$, with $X_{s+\frac{1}{2}}^{\left(e_{j}\right)}=\frac{X_{s+1}^{\left(e_{j}\right)}+X_{s}^{\left(e_{j}\right)}}{2}$. Let $\tilde{K}_{q}^{(\sigma)}$ as defined in (3.26). Then, the finite difference scheme is equivalent to the continuity condition 1.10 and Dirichlet condition for the pressure 1.11) coupled with following equations:

$$
\begin{aligned}
& U_{s+\frac{1}{2}, q}^{\left(e_{j}\right)} \in S_{H}^{\left(\sigma_{j}\right)} \subset L^{2}\left(\sigma_{j}\right) \text { if } 1 \leq j \leq M, 0 \leq s<S^{\left(e_{j}\right)}, 0 \leq q<Q, \\
& U_{s, 0}^{\left(e_{j}\right)}=0 \text { if } 1 \leq j \leq M, 0 \leq s<S^{\left(e_{j}\right)}, \\
& U_{s+\frac{1}{2}, q+1}^{\left(e_{j}\right)}=\left(I-k\left(T_{H}^{\left(\sigma_{j}\right)}\right)^{-1}\right)^{-1} U_{s+\frac{1}{2}, q+1}^{\left(e_{j}\right)}-k \frac{P_{s+1, q+1}^{\left(e_{j}\right)}-P_{s, q+1}^{\left(e_{j}\right)}}{h^{\left(e_{j}\right)}} W^{\left(\sigma_{j}\right)} \text { if }\left\{\begin{array}{l}
1 \leq j \leq M, \\
0 \leq s<S^{\left(e_{j}\right)}, \\
0 \leq q<Q,
\end{array},\right. \\
& f_{s, q+1}^{\left(e_{j}\right)}=\int_{\sigma_{j}} \frac{U_{s+\frac{1}{2}, q+1}^{\left(e_{j}\right)}-U_{s-\frac{1}{2}, q+1}^{\left(e_{j}\right)}}{h^{\left(e_{j}\right)}} \mathrm{d} x \quad \text { if } 1 \leq j \leq M, 1<s<S^{\left(e_{j}\right)}, 0 \leq q<Q, \\
& \Psi_{\ell, q+1}=\sum_{1 \leq j \leq M, O_{\ell} \in e_{j}}\left(-\frac{h^{\left(e_{j}\right)}}{2} f_{s, q+1}^{\left(e_{j}\right)}+\alpha_{\ell, j} \int_{\sigma_{j}} U_{s+\frac{1}{2} \alpha_{\ell, j}, q+1}^{\left(e_{j}\right)} \mathrm{d} x\right) \quad \text { if } 1 \leq \ell \leq N, 0 \leq q<Q,
\end{aligned}
$$

where, in the sum, $s$ is such that $X_{s}^{\left(e_{j}\right)}=O_{\ell}$.

This formulation can be solved in the following way. When the $U_{s, q}^{\left(e_{j}\right)}$ are known for a given $q \in$ $\{0,1 \ldots, Q-2\}, P_{s, q+1}^{\left(e_{j}\right)}$ is the unique solution to 1.10 -1.11 coupled with the system:

$$
\begin{array}{r}
k \tilde{K}_{0}^{(\sigma)} \frac{P_{s+1, q+1}^{\left(e_{j}\right)}-2 P_{s, q+1}^{\left(e_{j}\right)}+P_{s-1, q+1}^{\left(e_{j}\right)}}{\left(h^{\left(e_{j}\right)}\right)^{2}}=-f_{s, q+1}^{\left(e_{j}\right)}-\int_{\sigma_{j}}\left(I-k\left(T_{H}^{\left(\sigma_{j}\right)}\right)^{-1}\right)^{-1} \frac{U_{s+\frac{1}{2}, q}^{\left(e_{j}\right)}-U_{s-\frac{1}{2}, q}^{\left(e_{j}\right)} \mathrm{d} x}{h^{\left(e_{j}\right)}} \\
\text { if } 1 \leq j \leq M, 0 \leq s<S^{\left(e_{j}\right)}, 0 \leq q<Q, \\
\Psi_{\ell, q+1}=\sum_{1 \leq j \leq M, O_{\ell} \in e_{j}}\left(-\frac{h^{\left(e_{j}\right)}}{2} f_{s, q+1}^{\left(e_{j}\right)}-k \tilde{K}_{0}^{\left(\sigma_{j}\right)} \frac{P_{s+\alpha_{\ell, j}, q+1}^{\left(e_{j}\right)}-P_{s, q+1}^{\left(e_{j}\right)}}{h^{\left(e_{j}\right)}}\right. \\
\left.+\alpha_{\ell, j} \int_{\sigma_{j}}\left(I-k\left(T_{H}^{\left(\sigma_{j}\right)}\right)^{-1}\right)^{-1} U_{s+\frac{1}{2} \alpha_{\ell, j}, q}^{\left(e_{j}\right)} \mathrm{d} x\right), \text { if } 1 \leq \ell \leq N, 0 \leq q<Q,
\end{array}
$$

where in the sum, $s$ is such that $X_{s}^{\left(e_{j}\right)}=O_{\ell}$.

Once $\left(P_{s, q+1}^{\left(e_{j}\right)}\right)$ are known, $\left(U_{s+\frac{1}{2}, q+1}^{\left(e_{j}\right)}\right)$ are straightforward to compute.

Note that the system to inverse is the same as for the block triangular approach.

If we denote $R$ the time to compute the Choleski factorization of $\left(I-k\left(T_{H}^{(\sigma)}\right)^{-1}\right)$ and $r$ the number of non-zero elements of the corresponding factors, then the total cost is $O\left(N \mathbf{S}+N^{3}+N R+Q \mathbf{S}\left(N \mathbf{S}+N^{2}\right) r\right)$. 


\section{Convergence of the scheme}

The following theorem is the main result of this paper. It proves that the proposed scheme on the graph is convergent with order one in space and time, provided the discretization in the crosssection is well-chosen: either $\theta(k)=0$ (exact kernels) or the order is limited by the accuracy of the approximations of the kernels discussed above (3.27), 3.31).

With additional assumptions, we improve the order of convergence in Theorem 2.

Theorem 1. Let $\theta(k)=\max _{1 \leq j \leq M}\left|K_{0}^{\left(\sigma_{j}\right)}-\tilde{K}_{0}^{\left(\sigma_{j}\right)}\right|+\sum_{q=1}^{Q-1}\left|K_{q}^{\left(\sigma_{j}\right)}-K_{q-1}^{\left(\sigma_{j}\right)}-\tilde{K}_{q}^{\left(\sigma_{j}\right)}+\tilde{K}_{q-1}^{\left(\sigma_{j}\right)}\right|$.

Let $\mathbf{p}_{h, k} \in L^{2}\left(0, T, H^{1}(\mathcal{B})\right)$ be the function such that:

- $\mathbf{p}_{h, k}$ is affine on $\left[X_{s}^{\left(e_{j}\right)}, X_{s+1}^{\left(e_{j}\right)}\right] \times\{t\}$ for $t \in[0, T], 1 \leq j \leq M, s \in\left\{0, \ldots, S^{\left(e_{j}\right)}-1\right\}$.

- $\mathbf{p}_{h, k}\left(X_{s}^{\left(e_{j}\right)}, t\right)=P_{s, q}^{\left(e_{j}\right)}$ for $\left.t \in\right] t_{q}, t_{q+1}\left[, 1 \leq j \leq M, s \in\left\{0, \ldots, S^{\left(e_{j}\right)}\right\}, q \in\{0, \ldots, Q-1\}\right.$.

Assume that

$$
\theta(k) \rightarrow 0 \text { as } k \rightarrow 0, F \in H_{00}^{1}\left(0, T, H_{d c}^{1}(\mathcal{B})\right), \Psi_{1}, \ldots, \Psi_{N} \in H_{00}^{1}(0, T) .
$$

Then:

$$
\mathbf{p}_{h, k} \rightarrow P \text { in } L^{2}\left(0, T, H^{1}(\mathcal{B})\right) \text { when }(h, k) \rightarrow(0,0) .
$$

Furthermore, there exists $C_{1}, C_{2}$ such that if:

$$
\theta(k)<C_{2}, F \in H_{00}^{2}\left(0, T, H_{d c}^{2}(\mathcal{B})\right), \Psi_{1}, \ldots, \Psi_{N} \in H_{00}^{2}(0, T),
$$

then $P \in H^{1}\left(0, T, H_{d c}^{2}(\mathcal{B})\right)$ and:

$$
\begin{aligned}
\left\|\mathbf{p}_{h, k}-P\right\|_{L^{2}\left(0, T, H^{1}(\mathcal{B})\right)} \leq & C_{1}\left(h\|P\|_{L^{2}\left(0, T, H_{d c}^{2}(\mathcal{B})\right)}+\frac{h}{C_{2}-\theta(k)}\|F\|_{H^{1}\left(0, T, H_{d c}^{1}(\mathcal{B})\right)}\right. \\
& \left.+k\|P\|_{H^{1}\left(0, T, H^{1}(\mathcal{B})\right)}+\|P\|_{L^{2}\left(0, T, H^{1}(\mathcal{B})\right)} \frac{\theta(k)}{C_{2}-\theta(k)}\right) .
\end{aligned}
$$

Proof: We use the following lemma.

Lemma 5. There exists $C>0$ such that, for every $u \in H^{1}\left(0, T, H^{1}(\mathcal{B}) \cap H_{d c}^{2}(\mathcal{B})\right)$ :

$$
\inf _{\psi \in \mathbb{P}_{k}^{0}\left(0, T, \mathbb{P}_{h}^{1}(\mathcal{B})\right)}\|\psi-u\|_{L^{2}\left(0, T, H^{1}(\mathcal{B})\right)} \leq C\left(h\|u\|_{L^{2}\left(0, T, H_{d c}^{2}(\mathcal{B})\right)}+k\|u\|_{H^{1}\left(0, T, H^{1}(\mathcal{B})\right)}\right)
$$

Proof of the Lemma: If $u \in H^{1}(\mathcal{B}) \cap H_{d c}^{2}(\mathcal{B})$ and $P_{h}$ is the continuous piecewise linear interpolant in $\mathbb{P}_{h}^{1}(\mathcal{B})$ at the points of the subdivision, then:

$$
\left\|u-P_{h} u\right\|_{H^{1}(\mathcal{B})} \leq C h\|u\|_{H_{d c}^{2}(\mathcal{B})}, \quad\left\|P_{h} u\right\|_{H^{1}(\mathcal{B})} \leq C\|u\|_{H^{1}(\mathcal{B})} .
$$

Now, let $v \in H^{1}(0, T)$ and let $Q_{k}$ be the $L^{2}$-projector on $\mathbb{P}_{k}^{0}(0, T)$. Then:

$$
\left\|v-Q_{k} v\right\|_{L^{2}(\mathcal{B})} \leq C k\|v\|_{H^{1}(\mathcal{B})}
$$

and

$$
\begin{array}{r}
\left\|p-P_{h} Q_{k} p\right\|_{L^{2}\left(0, T, H^{1}(\mathcal{B})\right)} \leq\left\|p-Q_{k} p\right\|_{L^{2}\left(0, T, H^{1}(\mathcal{B})\right)}+\left\|Q_{k} p-P_{h} Q_{k} p\right\|_{L^{2}\left(0, T, H^{1}(\mathcal{B})\right)} \\
\leq C k\|p\|_{H^{1}\left(0, T, H^{1}(\mathcal{B})\right)}+C h\left\|Q_{k} p\right\|_{L^{2}\left(0, T, H_{d c}^{2}(\mathcal{B})\right)} \\
\leq C k\|p\|_{H^{1}\left(0, T, H^{1}(\mathcal{B})\right)}+C h\|p\|_{L^{2}\left(0, T, H_{d c}^{2}(\mathcal{B})\right)} .
\end{array}
$$

This proves the lemma.

Now, we prove the convergence theorem. 
Let $\alpha$ be the coercivity constant of $a_{T}$. From Proposition 1 in Section 3.2 we know that:

$$
\left\|a_{T, k}-a_{T}\right\| \leq \theta(k) .
$$

Thus, for $k$ small enough, $a_{T, k}$ is $(\alpha-\theta(k))$-coercive. Let $\alpha(k)$ be the constant of coercivity of $a_{T, k}$. Let $p_{h, k} \in \mathbb{P}_{k}^{0}\left(0, T, \mathbb{P}_{h}^{1}(\mathcal{B})\right)$ be the unique solution of:

$$
\forall \psi \in \mathbb{P}_{k}^{0}\left(0, T, \mathbb{P}_{h}^{1}(\mathcal{B})\right), \quad a_{T}\left(p_{h, k}, \psi\right)=b_{T}(\psi) .
$$

According to Céa's lemma, we have:

$$
\left\|P-p_{h, k}\right\|_{L^{2}\left(0, T, H^{1}(\mathcal{B})\right)} \leq \frac{\left\|a_{T}\right\|}{\alpha} \inf _{\psi \in \mathbb{P}_{k}^{0}\left(0, T, \mathbb{P}_{h}^{1}(\mathcal{B})\right)}\|P-\psi\|_{L^{2}\left(0, T, H^{1}(\mathcal{B})\right)} .
$$

Let us now estimate $p_{h, k}-\mathbf{p}_{h, k}$. Using (3.19), we have:

$$
a_{T, k}\left(\mathbf{p}_{h, k}-p_{h, k}, \psi\right)=a_{T}\left(p_{h, k}, \psi\right)-a_{T, k}\left(p_{h, k}, \psi\right)+b_{T, h}(\psi)-b_{T}(\psi) .
$$

By taking $\psi=p_{h, k}-\mathbf{p}_{h, k}$ and using coercivity on the left-hand side and continuity on the right-hand side, we get:

$$
\alpha(k)\left\|p_{h, k}-\mathbf{p}_{h, k}\right\|_{L^{2}\left(0, T, H^{1}(\mathcal{B})\right)} \leq \theta(k)\left\|p_{h, k}\right\|+\left\|b_{T, h}-b_{T}\right\|_{L^{2}\left(0, T, H^{1}(\mathcal{B})\right)^{\prime}} .
$$

Hence, we have:

$$
\begin{aligned}
& \left\|P-\mathbf{p}_{h, k}\right\|_{L^{2}\left(0, T, H^{1}(\mathcal{B})\right)} \\
& \leq \frac{\left\|a_{T}\right\|}{\alpha} \inf _{\psi \in \mathbb{P}_{k}^{0}\left(0, T, \mathbb{P}_{h}^{1}(\mathcal{B})\right)}\|P-\psi\|_{L^{2}\left(0, T, H^{1}(\mathcal{B})\right)} \\
& +\frac{\theta(k)}{\alpha(k)}\left\|p_{h, k}\right\|_{L^{2}\left(0, T, H^{1}(\mathcal{B})\right)}+\frac{1}{\alpha(k)}\left\|b_{T, h}-b_{T}\right\|_{L^{2}\left(0, T, H^{1}(\mathcal{B})\right)^{\prime}}
\end{aligned}
$$

Let us now show that $\lim _{(h, k) \rightarrow(0,0)}\left\|P-\mathbf{p}_{h, k}\right\|_{L^{2}\left(0, T, H^{1}(\mathcal{B})\right)}=0$. As $H^{1}\left(0, T, H^{1}(\mathcal{B}) \cap H_{d c}^{2}((\mathcal{B}))\right.$ is dense in $L^{2}\left(0, T, H^{1}((\mathcal{B}))\right.$, Lemma 5 implies that

$$
\lim _{(h, k) \rightarrow(0,0)} \inf _{\psi \in \mathbb{P}_{k}^{0}\left(0, T, \mathbb{P}_{h}^{1}(\mathcal{B})\right)}\|P-\psi\|_{L^{2}\left(0, T, H^{1}(\mathcal{B})\right)}=0 .
$$

From equation 3.20, $\lim _{h \rightarrow 0}\left\|b_{T, h}-b_{T}\right\|_{L^{2}\left(0, T, H^{1}(\mathcal{B})\right)^{\prime}}=0$, and, as $\alpha(k) \geq \alpha-\theta(k)$ implies that $\lim \inf \alpha(k)>0$, we conclude that

$$
\lim _{(h, k) \rightarrow(0,0)}\left\|P-\mathbf{p}_{h, k}\right\|_{L^{2}\left(0, T, H^{1}(\mathcal{B})\right)}=0 .
$$

This is the first part of Theorem 1. Now, if we assume in addition that $F \in H_{00}^{2}\left(0, T, H_{d c}^{2}(\mathcal{B})\right)$, $\Psi_{1}, \ldots, \Psi_{N} \in H_{00}^{2}(0, T)$, then, from the regularity resulsts of Section 2.3 , we have that $P \in$ $H_{00}^{1}\left(0, T, H_{d c}^{2}(\mathcal{B})\right)$. Hence, using Lemma 5 :

$$
\inf _{\psi \in \mathbb{P}_{k}^{0}\left(0, T, \mathbb{P}_{h}^{1}(\mathcal{B})\right)}\|P-\psi\|_{L^{2}\left(0, T, H^{1}(\mathcal{B})\right)} \leq C h\|P\|_{L^{2}\left(0, T, H_{d c}^{2}(\mathcal{B})\right)}+C k\|P\|_{H^{1}\left(0, T, H^{1}(\mathcal{B})\right)} .
$$

Besides, if $p_{h, k} \neq 0, \alpha\left\|p_{h, k}\right\|_{L^{2}\left(0, T, H^{1}(\mathcal{B})\right)} \leq \frac{1}{\left\|p_{h, k}\right\|_{L^{2}\left(0, T, H^{1}(\mathcal{B})\right)}} a_{T}\left(P, p_{h, k}\right) \leq\left\|a_{T}\right\|\|P\|_{L^{2}\left(0, T, H^{1}(\mathcal{B})\right)}$ and from equation $(3.20)$, we get:

$$
\left\|b_{T, h}-b_{T}\right\|_{L^{2}\left(0, T, H^{1}(\mathcal{B})\right)^{\prime}} \leq 3 h\|F\|_{H^{1}\left(0, T, H_{d c}^{1}(\mathcal{B})\right)} .
$$

Also, $\alpha(k) \geq \alpha-\theta(k)$. Combining all these inequalities into 4.33, we get the second part of Theorem 1 .

Let us introduce the following lemma which is used to prove Theorem 2. 
Lemma 6. Let $p:[0, T] \rightarrow \mathbb{R}$ a $C^{2}$ function. Let $\tilde{p}=\sum_{q=0}^{Q-1} p\left(t_{q+1 / 2}\right) \phi_{q}$, where $\phi_{q}=\mathbf{1}_{\left[t_{q}, t_{q+1}[\right.}$.

Assume that $K:[0, T] \rightarrow \mathbb{R}$ is a continuous function, continuously differentiable on $] 0, T]$, such that $K \geq 0, K^{\prime} \leq 0$ and that $K^{\prime}(t)=O_{t \rightarrow 0^{+}}\left(t^{-1 / 2}\right)$. Let $L p(t)=\int_{0}^{t} K(t-s) p(s) \mathrm{d} s$.

Then, for any $\phi \in \mathbb{P}_{k}(0, T)$ :

$$
\left|\int_{0}^{T}(L(p-\tilde{p}))^{\prime}(t) \phi(t) \mathrm{d} t\right| \leq C k^{2}\left(\left\|p^{\prime}\right\|_{\infty} \sqrt{\log (T / k)}+\left\|p^{\prime \prime}\right\|_{\infty}\right)\|\phi\|_{L^{2}} .
$$

If furthermore $p^{\prime}(0)=0$, then:

$$
\left|\int_{0}^{T}(L(p-\tilde{p}))^{\prime}(t) \phi(t) \mathrm{d} t\right| \leq C k^{2}\left\|p^{\prime \prime}\right\|_{\infty}\|\phi\|_{L^{2}} .
$$

\section{Proof:}

Let $\mu(t)=\int_{k / 2}^{t}(p-\tilde{p})(\tau) \mathrm{d} \tau$, so that $\mu$ is $C^{2}$ on $[0, T] \backslash\left\{t_{0}, t_{1}, \ldots, t_{Q}\right\}$ and $\mu^{\prime}\left(t_{q+1 / 2}\right)=0$ for $q \in\{0, \ldots, Q-1\}$.

Besides, for any $t \in] 0, T-k \llbracket \backslash\left\{t_{0}, t_{1}, \ldots, t_{Q}\right\}$, there exists $q \in\{0,1, \ldots, Q\}$ such that $\left.t_{q} \in\right] t, t+k[$. Then:

$$
\left|\mu^{\prime \prime}(t+k)-\mu^{\prime \prime}(t)\right|=\left|p^{\prime}(t+k)-p^{\prime}(t)\right| \leq k\left\|p^{\prime \prime}\right\|_{\infty} .
$$

Integrating from $s=t_{q-1 / 2}$ to $s=t$, we get: $\left|\mu^{\prime}(t+k)-\mu^{\prime}(t)\right| \leq k^{2}\left\|p^{\prime \prime}\right\|_{\infty}$.

Integrating from $s=t_{q-1}$ to $s=t$, using $\left|\mu\left(t_{q}\right)-\mu\left(t_{q}-k\right)\right|=\left|\int_{t_{q-1}}^{t_{q}} p(t)-p\left(t_{q+1 / 2}\right) \mathrm{d} t\right| \leq k^{3}\left\|p^{\prime \prime}\right\|_{\infty}$, we get:

$$
|\mu(t+k)-\mu(t)| \leq k^{3}\left\|p^{\prime \prime}\right\|_{\infty}+\left|\mu\left(t_{q}\right)-\mu\left(t_{q}-k\right)\right| \leq 2 k^{3}\left\|p^{\prime \prime}\right\|_{\infty}
$$

Let us now introduce $\mu$ in the integral to be bounded:

$$
\begin{aligned}
\int_{0}^{T}(L(p-\tilde{p}))^{\prime}(t) \phi_{q}(t) \mathrm{d} t= & \int_{t_{q}}^{t_{q+1}}\left(K(0)(p-\tilde{p})(t)+\int_{0}^{t} K^{\prime}(\tau)(p-\tilde{p})(t-\tau) \mathrm{d} \tau\right) \mathrm{d} t \\
= & K(0)\left(\mu\left(t_{q+1}\right)-\mu\left(t_{q}\right)\right)+\int_{t_{q}}^{t_{q+1}} \int_{0}^{t_{q}} K^{\prime}(\tau)(p-\tilde{p})(t-\tau) \mathrm{d} \tau \mathrm{d} t \\
& +\int_{t_{q}}^{t_{q+1}} \int_{t_{q}}^{t} K^{\prime}(\tau)(p-\tilde{p})(t-\tau) \mathrm{d} \tau \mathrm{d} t \\
= & K(0)\left(\mu\left(t_{q+1}\right)-\mu\left(t_{q}\right)\right)+\int_{0}^{t_{q}} K^{\prime}(\tau)\left(\mu\left(t_{q+1}-\tau\right)-\mu\left(t_{q}-\tau\right)\right) \mathrm{d} \tau \\
& +\int_{t_{q}}^{t_{q+1}} \int_{t_{q}}^{t} K^{\prime}(\tau)(p-\tilde{p})(t-\tau) \mathrm{d} \tau \mathrm{d} t
\end{aligned}
$$

Since $-C t^{-\frac{1}{2}} \leq K^{\prime}(t) \leq 0$ and thus $\int_{0}^{T}\left|K^{\prime}(t)\right| \mathrm{d} t \leq K(0)$, and since $\|p-\tilde{p}\| \leq k\left\|p^{\prime}\right\|$, we get:

$$
\begin{aligned}
\left|\int_{0}^{T}(L(p-\tilde{p}))^{\prime}(t) \phi_{q}(t) \mathrm{d} t\right| & \leq K(0) k^{3}\left\|p^{\prime \prime}\right\|_{\infty}+2 K(0) k^{3}\left\|p^{\prime \prime}\right\|_{\infty}+k\left\|p^{\prime}\right\|_{\infty} \int_{t_{q}}^{t_{q+1}} \int_{t_{q}}^{t} C t^{-\frac{1}{2}} \mathrm{~d} \tau \mathrm{d} t \\
& \leq 3 K(0) k^{3}\left\|p^{\prime \prime}\right\|_{\infty}+2 C k^{\frac{5}{2}}(q+1)^{-\frac{1}{2}}\left\|p^{\prime}\right\|_{\infty} .
\end{aligned}
$$

From this, we conclude that, for $\phi=\sum_{q=0}^{Q-1} a_{q} \phi_{q} \in \mathbb{P}_{k}^{0}(0, T)$ :

$$
\left|\int_{0}^{T}(L(p-\tilde{p}))^{\prime}(t) \phi(t) \mathrm{d} t\right| \leq 2 \sum_{q=0}^{Q-1}\left|a_{q}\right| C(q+1)^{-\frac{1}{2}} k^{\frac{5}{2}}\left\|p^{\prime}\right\|_{\infty}+3 \sum_{q=0}^{Q-1}\left|a_{q}\right| k^{3} K(0)\left\|p^{\prime \prime}\right\|_{\infty} .
$$


With Cauchy-Schwarz inequality this yields:

$$
\begin{gathered}
\left|\int_{0}^{T}(L(p-\tilde{p}))^{\prime}(t) \phi(t) \mathrm{d} t\right| \leq 2 C \frac{\|\phi\|_{L^{2}}}{\sqrt{k}} k^{\frac{5}{2}}\left\|p^{\prime}\right\|_{\infty}\left(\sum_{q=0}^{Q-1}(q+1)^{-1}\right)^{1 / 2}+3 \frac{\|\phi\|_{L^{2}}}{\sqrt{k}} \sqrt{\frac{T}{k}} K(0) k^{3}\left\|p^{\prime \prime}\right\|_{\infty}, \\
\left|\int_{0}^{T}(L(p-\tilde{p}))^{\prime}(t) \phi(t) \mathrm{d} t\right| \leq\left(2 C k^{2}\left\|p^{\prime}\right\|_{\infty}(\log (T / k))^{1 / 2}+3 \sqrt{T} K(0) k^{2}\left\|p^{\prime \prime}\right\|_{\infty}\right)\|\phi\|_{L^{2}} .
\end{gathered}
$$

If furthermore $p^{\prime}(0)=0$, then, for $t \in[0, k],|(p-\tilde{p})(t)| \leq k^{2}\left\|p^{\prime \prime}\right\|_{\infty}$. We conclude that, in this case:

$$
\begin{aligned}
\left|\int_{0}^{T}(L(p-\tilde{p}))^{\prime}(t) \phi_{q}(t) \mathrm{d} t\right| & \leq 3 K(0) k^{3}\left\|p^{\prime \prime}\right\|_{\infty}+k^{2}\left\|p^{\prime \prime}\right\|_{\infty} \int_{t_{q}}^{t_{q+1}} \int_{t_{q}}^{t}\left|K^{\prime}(\tau)\right| \mathrm{d} \tau \mathrm{d} t \\
& \leq 3 K(0) k^{3}\left\|p^{\prime \prime}\right\|_{\infty}+K(0) k^{3}\left\|p^{\prime \prime}\right\|_{\infty}
\end{aligned}
$$

Hence:

$$
\left|\int_{0}^{T}(L(p-\tilde{p}))^{\prime}(t) \phi(t) \mathrm{d} t\right| \leq 4 K(0) \sqrt{T} k^{2}\|\phi\|_{L^{2}} .
$$

This ends the proof of Lemma 6 . Now, we prove Theorem 2 below.

Theorem 2. Let $\theta(k)$ and $\mathbf{p}_{h, k}$ be defined as in Theorem 1 .

Let $\tilde{p}_{h, k} \in L^{2}\left(0, T, H^{1}(\mathcal{B})\right)$ be the function such that:

- $\tilde{p}_{h, k}$ is affine on $\left[X_{s}^{\left(e_{j}\right)}, X_{s+1}^{\left(e_{j}\right)}\right] \times\{t\}$ for $t \in[0, T], 1 \leq j \leq M, s \in\left\{0, \ldots, S^{\left(e_{j}\right)}-1\right\}$.

- $\tilde{p}_{h, k}\left(X_{s}^{\left(e_{j}\right)}, t\right)=P\left(X_{s}^{\left(e_{j}\right)}, t_{q+1 / 2}\right)$ for $\left.t \in\right] t_{q}, t_{q+1}\left[, 1 \leq j \leq M, s \in\left\{0, \ldots, S^{\left(e_{j}\right)}\right\}, q \in\right.$ $\{0, \ldots, Q-1\}$.

Assume that the kernels $K^{(\sigma)}$ satisfy the hypotheses of Lemma 6; assume that $P$ is a $C^{4}$ function on each edge of the graph;

assume that $\theta(k) \rightarrow 0$ as $k \rightarrow 0$.

Let $\beta(k)$ be defined by $\beta(k)=k^{2}$ if $\frac{\partial P}{\partial t}(\cdot, 0)$ is constant, $\left.\beta(k)=k^{2} \sqrt{\log (T / k}\right)$ otherwise.

Then, there exists two positive constants $C_{1}, C_{2}$ such that for $\theta(k)<C_{2}$ :

$$
\left\|\mathbf{p}_{h, k}-\tilde{p}_{h, k}\right\|_{L^{2}\left(0, T, H^{1}(\mathcal{B})\right)} \leq \frac{C_{1}}{C_{2}-\theta(k)}\left(\beta(k)+h^{2}+\theta(k)\right) m(P),
$$

where $m(P)=\left\|\frac{\partial^{2} P}{\partial x \partial t}\right\|_{\infty}+\left\|\frac{\partial^{3} P}{\partial x \partial t^{2}}\right\|_{\infty}+\left\|\frac{\partial^{4} P}{\partial x^{4}}\right\|_{\infty}$.

Remark The fact that $K^{(\sigma)}$ satisfies the assumptions of Lemma 6 for any smooth $\sigma$ and also for all the examples given in Section 3.4.1 is proven in [8].

Proof

Let $\tilde{p}_{h}(t, x)=\sum_{X \in \mathcal{P}_{h}} P(X, t) \psi^{X}$. Since from equation 1.2

$$
\frac{\partial F}{\partial t}(x, t)=-K^{(\bar{\sigma})}(0) \frac{\partial^{2} P}{\left(\partial x^{(\bar{e})}\right)^{2}}(x, t)-\int_{0}^{t}\left(K^{(\bar{\sigma})}\right)^{\prime}(t-\tau) \frac{\partial^{2} P}{\left(\partial x^{(\bar{e})}\right)^{2}}(x, \tau) \mathrm{d} \tau,
$$

we have that $F \in H_{00}^{1}\left(0, T, H_{d c}^{2}(\mathcal{B})\right)$ and that $\|F\|_{H^{1}\left(0, T, H_{d c}^{2}(\mathcal{B})\right)} \leq C m(P)$. Similarly, $\Psi_{1}, \ldots, \Psi_{n} \in$ $H_{00}^{1}(0, T)$.

Let $\phi \in \mathbb{P}_{k}^{0}(0, T)$ and $\psi \in \mathbb{P}_{h}^{1}(\mathcal{B})$. From the previous lemma, it follows that:

$$
\left|a_{T}\left(p_{h, k}-\tilde{p}_{h}, \phi \psi\right)\right| \leq C\|\phi\|_{H^{1}}\|\psi\|_{L^{2}} \beta(k) m(P)
$$


where $a_{T}$ is defined in 2.15 . Let us compute now $a_{T}\left(P-\tilde{p}_{h}, \phi \psi\right)$. As

$$
\begin{aligned}
\int_{\left[X_{s}^{(e)}, X_{s+1}^{(e)}\right]} \frac{\partial^{2} L^{(\bar{\sigma})}\left(P-\tilde{p}_{h}\right)}{\partial t \partial x^{(e)}} \frac{\partial \psi}{\partial x^{(e)}} \mathrm{d} x= & \left(\frac{\partial\left(L^{\bar{\sigma}} P\right)}{\partial t}\left(X_{s+1}^{(e)}, t\right)-\frac{\partial\left(L^{\bar{\sigma}} P\right)}{\partial t}\left(X_{s}^{(e)}, t\right)\right. \\
& \left.-\frac{\partial\left(L^{(\bar{\sigma})} \tilde{p}_{h}\right)}{\partial t}\left(X_{s+1}^{(e)}, t\right)+\frac{\partial\left(L^{(\bar{\sigma})} \tilde{p}_{h}\right)}{\partial t}\left(X_{s}^{(e)}, t\right)\right) \frac{\partial \psi}{\partial x^{(e)}}\left(X_{s+\frac{1}{2}}^{(e)}\right) h^{(e)}, \\
= & 0
\end{aligned}
$$

we get that $a_{T}\left(P-\tilde{p}_{h}, \phi \psi\right)=0$, so that

$$
\left|a_{T}\left(P-\tilde{p}_{h, k}, \phi \psi\right)\right| \leq C \beta(k)\|\phi\|_{H^{1}}\|\psi\|_{L^{2}} m(P) .
$$

Hence, for any $\psi \in \mathbb{P}_{k}^{0}\left(0, T, \mathbb{P}_{h}^{1}(\mathcal{B})\right)$ :

$$
\left|a_{T}\left(P-\tilde{p}_{h, k}, \psi\right)\right| \leq C \beta(k)\|\psi\|_{L^{2}\left(0, T, H^{1}(\mathcal{B})\right)} m(P) .
$$

We have:

$$
\begin{aligned}
a_{T, k}\left(\mathbf{p}_{h, k}-\tilde{p}_{h, k}, \psi\right) & =a_{T, k}\left(\mathbf{p}_{h, k}, \psi\right)+\left(a_{T}-a_{T, k}\right)\left(\tilde{p}_{h, k}, \psi\right)+a_{T}\left(P-\tilde{p}_{h, k}, \psi\right)-a_{T}(P, \psi) \\
& =\left(b_{T, h}-b_{T}\right)(\psi)+\left(a_{T}-a_{T, k}\right)\left(\tilde{p}_{h, k}, \psi\right)+a_{T}\left(P-\tilde{p}_{h, k}, \psi\right)
\end{aligned}
$$

where $a_{T, k}$ is defined in (3.18). Taking $\psi=\mathbf{p}_{h, k}-\tilde{p}_{h, k}$, using the coercivity of $a_{T, k}$ on the left handside, using continuity of $a_{T}, a_{T}-a_{T, k}$ and the previous inequality on the right handside, and dividing by the norm of $\mathbf{p}_{h, k}-\tilde{p}_{h, k}$, we get:

$$
\alpha(k)\left\|\mathbf{p}_{h, k}-\tilde{p}_{h, k}\right\|_{L^{2}\left(0, T, H^{1}(\mathcal{B})\right)}^{2-1} \leq C h^{2}\|F\|_{H^{1}\left(0, T, H_{d c}^{2}(\mathcal{B})\right)}+\theta(k)\left\|\tilde{p}_{h, k}\right\|_{L^{2}\left(0, T, H^{1}(\mathcal{B})\right)}+C \beta(k) m(P) .
$$

With $\|F\|_{H^{1}\left(0, T, H_{d c}^{2}(\mathcal{B})\right)} \leq C m(P),\left\|p_{h, k}\right\|_{H^{1}\left(0, T, H_{d c}^{2}(\mathcal{B})\right)} \leq C m(P)$ and $\alpha(k) \geq\left\|a_{T}\right\|-\theta(k)$, the proof is complete.

Corollary 1 If $P$ is of class $C^{4}$, then:

$$
\left\|\tilde{p}_{h, k}-\mathbf{p}_{h, k}\right\|_{L^{\infty}\left(0, T, H^{1}(\mathcal{B})\right)} \leq C m(P)\left(\theta(k) k^{-\frac{1}{2}}+h^{2} k^{-\frac{1}{2}}+k^{\frac{3}{2}} \log (T / k)\right) .
$$

If $P$ is of class $C^{5}$ and $\frac{\partial^{r} P}{\partial t^{r}}(\cdot, 0)=0$ for $r \in\{0, \ldots, 5\}$, then:

$$
\left\|\tilde{p}_{h, k}-\mathbf{p}_{h, k}\right\|_{L^{\infty}\left(0, T, H^{1}(\mathcal{B})\right)} \leq C m\left(\frac{\partial P}{\partial t}\right)\left(\theta(k)+h^{2}+k^{2}\right)
$$

Proof: The first part is obtained by using that, for $\phi \in \mathbb{P}^{0}(0, T)$ :

$$
\sqrt{k}\|\phi\|_{L^{\infty}(0, T)} \leq\|\phi\|_{L^{2}(0, T)} .
$$

Hence:

$$
\sqrt{k}\left\|\mathbf{p}_{h, k}-\tilde{p}_{h, k}\right\|_{L^{\infty}\left(0, T, H^{1}(\mathcal{B})\right)} \leq\left\|\mathbf{p}_{h, k}-\tilde{p}_{h, k}\right\|_{L^{2}\left(0, T, H^{1}(\mathcal{B})\right)},
$$

and Theorem 2 yields the result.

For the second part of the corollary, let us extend $P, \mathbf{p}_{h, k}, \tilde{p}_{h, k}$ by zero for negative times. Let us denote $\Delta_{k} \phi(t)=\frac{\phi(t)-\phi(t-k)}{k}$. Then $\Delta_{k} P$ is a solution to 1.2 with $F, \Psi_{1}, \ldots, \Psi_{N}$ replaced by $\Delta_{k} F, \Delta_{k} \Psi_{1}, \ldots, \Delta_{k} \Psi_{N}$. Applying Theorem 2 to $\Delta_{k} P$ over $[0, T]$, we get for sufficiently small $k$ :

$$
\left\|\Delta_{k} \mathbf{p}_{h, k}-\Delta_{k} \tilde{p}_{h, k}\right\|_{L^{2}\left(0, T, H^{1}(\mathcal{B})\right)} \leq C m\left(\Delta_{k} P\right)\left(\theta(k)+h^{2}+k^{2}\right) .
$$

But $m\left(\Delta_{k} P\right) \leq m\left(\frac{\partial P}{\partial t}\right)$, and for $\phi \in \mathbb{P}^{0}(0, T)$ extended by zero, $\|\phi\|_{L^{\infty}(0, T)} \leq \sqrt{T}\left\|\Delta_{k} \phi\right\|_{L^{2}(0, T)}$. Hence:

$$
\left\|\mathbf{p}_{h, k}-\tilde{p}_{h, k}\right\|_{L^{\infty}\left(0, T, H^{1}(\mathcal{B})\right)} \leq C m\left(\frac{\partial P}{\partial t}\right)\left(\theta(k)+h^{2}+k^{2}\right) .
$$

This completes the proof of the corollary. 


\section{$5 \quad$ Numerical results}

In Section 5.1, we test the numerical accuracy of the scheme 3.4 .2 with the variants in the cross-sections. In Section 5.2, we then compare the asymptotic model $(1.2)$ with the initial 3DNavier-Stokes equations.

Let us assume that an error $E$ converges to 0 when a parameter $\varepsilon$ tends to 0 and that $E \sim_{\varepsilon \rightarrow 0} C \varepsilon^{\alpha}$ for some constants $C$ and $\alpha$. The parameter $\alpha$ is the order of convergence of $E$ with respect to $\varepsilon$. Let us consider a strictly decreasing sequence $\left(\varepsilon_{n}\right)$ which converges to 0 and let $E_{n}$ be the corresponding sequence of errors. Then,

$$
\alpha=\lim _{n \rightarrow+\infty} \frac{\log \left(\frac{E_{n-1}}{E_{n}}\right)}{\log \left(\frac{\varepsilon_{n-1}}{\varepsilon_{n}}\right)} .
$$

Hence, in all the tables below, when we report an error $\left(E_{n}\right)_{n}$ and a varying parameter $\left(\varepsilon_{n}\right)_{n}$, we also report $\frac{\log \left(\frac{E_{n-1}}{E_{n}}\right)}{\log \left(\frac{\varepsilon_{n-1}}{\varepsilon_{n}}\right)}$ on a column on the right to estimate the order of convergence.

\subsection{Numerical accuracy}

To evaluate the numerical accuracy of the scheme 3.4 .2 , we first build an exact test case solution to the asymptotic system 1.2 .

\subsubsection{Test case on a single tube}

Let $d \in\{2,3\}$. We consider the case of a single tube $(M=1)$ of length 1 with two extremities $O_{1}=(\hat{0}, 0), O_{2}=(\hat{0}, 1)\left(N_{1}=N=2\right)$. Let the cross-section of the tube be $\left.\sigma=\right]-\frac{1}{2}, \frac{1}{2}[$ if $d=2$, and $\sigma=\left\{x \in \mathbb{R}^{2} ;\|x\|_{2}<1,\right\}$ if $d=3$.

Let us take $P\left(\left(\hat{0}, x_{3}^{(e)}\right), t\right)=p\left(x_{3}^{(e)}, t\right)=\exp \left((1-t) x_{3}^{(e)}-\frac{\beta}{t}\right)$ where $\beta \in\{0,1\}$. When $\beta=1, P$ and all its time derivatives are zero when $t \rightarrow 0$.

Then, the flow at the left extremity $O_{1}$ of the pipe is given by:

$$
\Psi_{1}(t)=-\int_{0}^{t} K^{(\sigma)}(s)(1-(t-s)) \exp \left(-\frac{\beta}{t-s}\right) \mathrm{d} s
$$

At the right extremity $\mathrm{O}_{2}$ of the pipe, it is given by:

$$
\Psi_{2}(t)=\int_{0}^{t} K^{(\sigma)}(s)(1-(t-s)) \exp \left(1-\frac{\beta}{t-s}\right) \mathrm{d} s .
$$

The force applied along the pipe is:

$$
F\left(\left(\hat{0}, x_{3}^{(e)}\right), t\right)=-\int_{0}^{t} K^{(\sigma)}(s)(1-(t-s))^{2} \exp \left((1-(t-s)) x_{3}^{(e)}-\frac{\beta}{t-s}\right) \mathrm{d} s .
$$

The theoretical data $\Psi_{1}, \Psi_{2}, F$ can be numerically computed with a high accuracy by using the change of variable $\tau=\exp \left(1-\frac{1}{s}\right) t$ to remove the singularity at $s=0$. The integrand is then smooth and the Clenshaw-Curtis integration method yields accurate results.

For the sake of completeness, we chose to evaluate the numerical accuracy of the scheme with an approximate kernel $\left(\tilde{K}_{q}^{(\sigma)}\right)$ as proposed in Section 3.5.3 in the case $d=2$, while for the case $d=3$ we consider a tube with a disc cross-section and numerical approximation in the three following way: with the exact kernel given in Section 1.3, with a numerical approximation based on the discretization of equation (1.7), and last with this numerical approximation with a correction that uses the asympotic expansion 3.28 . 
Table 1: $\beta=1, p(x, t)=\mathrm{e}^{(1-t) x-1 /(t+\varepsilon)}$. In the last three lines, the order is computed with respect to $H$.

\begin{tabular}{|c|c|c|c|c|c|c|}
\hline$h$ & $k$ & $H$ & $\ell^{\infty}$-error $p_{x}$ & order & $\ell^{\infty}$-error $p$ & order \\
\hline \multirow{5}{*}{$2^{-15}$} & \multirow{5}{*}{$0.1 \cdot 2^{-12}$} & $2^{-2}$ & $4.78504 \mathrm{e}-06$ & & $3.13065 \mathrm{e}-06$ & \\
\hline & & $2^{-3}$ & $1.54869 \mathrm{e}-06$ & 1.63 & $1.02143 \mathrm{e}-06$ & 1.62 \\
\hline & & $2^{-4}$ & $2.56845 \mathrm{e}-07$ & 2.59 & $1.67585 \mathrm{e}-07$ & 2.61 \\
\hline & & $2^{-5}$ & $5.60860 \mathrm{e}-08$ & 2.20 & $3.90747 \mathrm{e}-08$ & 2.10 \\
\hline & & $2^{-6}$ & $1.10842 \mathrm{e}-07$ & -0.98 & $7.52349 \mathrm{e}-08$ & -0.95 \\
\hline \multirow{8}{*}{$2^{-13}$} & $0.1 \cdot 2^{-3}$ & \multirow{8}{*}{$2^{-6}$} & $3.96274 \mathrm{e}-05$ & & $2.62084 \mathrm{e}-05$ & \\
\hline & $0.1 \cdot 2^{-4}$ & & $2.30104 \mathrm{e}-05$ & 0.78 & $1.52338 \mathrm{e}-05$ & 0.78 \\
\hline & $0.1 \cdot 2^{-5}$ & & $1.24167 \mathrm{e}-05$ & 0.89 & $8.22755 \mathrm{e}-06$ & 0.89 \\
\hline & $0.1 \cdot 2^{-6}$ & & $6.45625 \mathrm{e}-06$ & 0.94 & $4.28260 \mathrm{e}-06$ & 0.94 \\
\hline & $0.1 \cdot 2^{-7}$ & & $3.29863 \mathrm{e}-06$ & 0.97 & $2.19189 \mathrm{e}-06$ & 0.97 \\
\hline & $0.1 \cdot 2^{-8}$ & & $1.67424 \mathrm{e}-06$ & 0.98 & $1.11614 \mathrm{e}-06$ & 0.97 \\
\hline & $0.1 \cdot 2^{-9}$ & & $8.50131 \mathrm{e}-07$ & 0.98 & $5.70325 \mathrm{e}-07$ & 0.97 \\
\hline & $0.1 \cdot 2^{-10}$ & & $4.33773 \mathrm{e}-07$ & 0.97 & $2.94556 \mathrm{e}-07$ & 0.95 \\
\hline $2^{-10}$ & $\frac{1}{1000}$ & $2^{-5}$ & $4.22920 \mathrm{e}-06$ & & $2.85494 \mathrm{e}-06$ & \\
\hline $2^{-12}$ & $\frac{1}{4000}$ & $2^{-6}$ & $1.08178 \mathrm{e}-06$ & 1.97 & $7.30885 \mathrm{e}-07$ & 1.97 \\
\hline $2^{-14}$ & $\overline{16000}$ & $2^{-7}$ & $2.72432 \mathrm{e}-07$ & 1.99 & $1.84098 \mathrm{e}-07$ & 1.99 \\
\hline
\end{tabular}

\subsubsection{Numerical accuracy when $d=2$}

For $d=2$, we test the scheme with the an approximate kernel $\left(\tilde{K}_{q}^{(\sigma)}\right)$ defined as proposed in Section 3.5.3.

Let $W(x)=1-\frac{\sinh \left(\left(\frac{1}{2}+x\right) / \sqrt{k}\right)+\sinh \left(\left(\frac{1}{2}-x\right) / \sqrt{k}\right)}{\sinh (1 / \sqrt{k})}$ be the solution of:

$$
\begin{cases}U-k U^{\prime \prime}=1 & \text { in } \sigma=]-1 / 2,1 / 2[, \\ U=0 & \text { on } \partial \sigma=\{-1 / 2,1 / 2\} .\end{cases}
$$

Let $H$ be a space step for the cross-section such that $1 / H$ is an integer. We consider the following difference scheme in the cross-section:

$$
\begin{cases}V_{0, r}=W\left(-\frac{1}{2}+r H\right) & \text { if } r \in\{1, \ldots, 1 / H-1\}, \\ \frac{V_{q, r}-V_{q+1}}{k}=\frac{V_{q, r-1}-2 V_{q, r}+V_{q, r-1}}{H^{2}} & \text { if } q \in\{1, \ldots, Q\}, r \in\{1, \ldots, 1 / H-1\}, \\ V_{q, 0}=V_{q, 1 / H}=0 & \text { if } q \in\{1, \ldots, Q\} .\end{cases}
$$

Then we take $\tilde{K}_{q}^{(\sigma)}=H \sum_{r=1}^{1 / H-1} V_{q, r}$.

We compute the maximum norm of the error of the numerical solution with respect to the exact solution. The results of the numerical test for different step sizes are listed in Table 1 when $\beta=1$ and in Table 2 when $\beta=0$.

We observe that the numerical order for the time step is 1 when $\beta=0$ and $1 / 2$ when $\beta=1$. Corollary 1 can be applied in both cases, but the accuracy is limited by the precision of the approximation of the kernel.

When $\beta=1$, we observe that the order of the error with respect to the cross-section space step $H$ is 2 . 
Table 2: $\beta=0, p(x, t)=\mathrm{e}^{(1-t) x}$. In the last three lines, the order is computed with respect to $H$.

\begin{tabular}{lllllll}
\hline$h$ & $k$ & $H$ & $\ell^{\infty}$-error $p_{x}$ & order & $\ell^{\infty}$-error $p$ & order \\
\hline \multirow{4}{*}{$0^{-13}$} & $0.1 \cdot 2^{-3}$ & & $2.23431 \mathrm{e}-01$ & & $1.37143 \mathrm{e}-01$ & \\
& $0.1 \cdot 2^{-4}$ & & $1.42094 \mathrm{e}-01$ & 0.65 & $8.75474 \mathrm{e}-02$ & 0.65 \\
& $0.1 \cdot 2^{-5}$ & & $9.28145 \mathrm{e}-02$ & 0.61 & $5.73905 \mathrm{e}-02$ & 0.61 \\
& $0.1 \cdot 2^{-6}$ & & $6.19066 \mathrm{e}-02$ & 0.58 & $3.83749 \mathrm{e}-02$ & 0.58 \\
& $0.1 \cdot 2^{-7}$ & $2^{-6}$ & $4.19479 \mathrm{e}-02$ & 0.56 & $2.60275 \mathrm{e}-02$ & 0.56 \\
& $0.1 \cdot 2^{-9}$ & & $2.87599 \mathrm{e}-02$ & 0.54 & $1.78284 \mathrm{e}-02$ & 0.55 \\
& $0.1 \cdot 2^{-10}$ & & $1.98894 \mathrm{e}-02$ & 0.53 & $1.22908 \mathrm{e}-02$ & 0.54 \\
\multirow{2}{*}{$2^{-10}$} & $\frac{1}{1000}$ & \multirow{2}{*}{$2^{-5}$} & $4.81106 \mathrm{e}-02$ & & $2.83381 \mathrm{e}-02$ & \\
$2^{-12}$ & $\frac{1}{4000}$ & $2^{-6}$ & $2.26623 \mathrm{e}-02$ & 1.09 & $1.38113 \mathrm{e}-02$ & 1.04 \\
$2^{-14}$ & $\frac{1}{16000}$ & $2^{-7}$ & $1.09880 \mathrm{e}-02$ & 1.04 & $6.81782 \mathrm{e}-03$ & 1.02 \\
\hline
\end{tabular}

\subsubsection{Numerical accuracy when $d=3$}

Since the cross-section is a disc, one could have used the radial symmetry to obtain a faster and more accurate scheme. However, the goal here is to obtain a benchmark for an arbitrary two-dimensional cross-section. Hence, we used unregular meshes for the disc.

In this section, we test the scheme 3.4 .2 with three different types of choice for $\left(\tilde{K}_{q}^{(\sigma)}\right)$ : the exact $\left(K_{q}^{(\sigma)}\right)$, and two approximations $\left(K_{\text {num }, q}^{(\sigma)}\right)$ and $\left(K_{\text {cor }, q}^{(\sigma)}\right)$ that are defined as in Section 3.4.3.

For these three approximations, we compute the $\ell^{\infty}$-error on $\frac{\partial P}{\partial x}$ and the results are reported in Table 4 for the case $\beta=0$, and Table 3 when $\beta=1$. The corresponding error curves are given in figure 2 .

The values of $\theta(k)$ for the numerical and corrected approximations will be studied in detail in the forthcoming second part of this article.

Let us summarize the orders with respect to $h, k, H$ that we have estimated:

\begin{tabular}{|c|c|c|c|c|}
\hline & & Numerical approximation & Corrected approximation & Exact \\
\hline & $h$ & 2 & 2 & 2 \\
\hline$\beta=0$ & $k$ & 0.5 & $\sim 1.4$ & $3 / 2$ \\
$\beta=1$ & $k$ & $\sim 1.6$ & $\sim 1.8$ & 2 \\
\hline$\beta=0$ & $H$ & 1 & $\sim 1.6$ & \\
$\beta=1$ & $H$ & $\sim 1.7$ & $\sim 1.7$ & \\
\hline
\end{tabular}

In all cases, the use of the exact kernel is the best option or close to it. When $\beta=1$, the three schemes yields similar accuracy if the edge space step $h$ in the cross-section space step $H$ is small enough. When $\beta=0$, the numerical approximation is slowly convergent and the corrected scheme offers a substantial improvement.

\subsection{Comparison with Navier-Stokes equations}

The aim of this section is to compare numerically the full 3D-Navier-Stokes equations (1.1) with the continuous asymptotic model on the graph 1.2 .

\subsubsection{Geometry and boundary condtions}

We do it for two different configurations, one constructed from a simply connected graph and the other one from a connected graph with a hole (see Figure 3).

In both cases, let $\Omega^{\varepsilon}$ be the interior of $\left\{M \in \mathbb{R}^{3} \mid \exists i, O \in e_{i}, O M \perp e_{i},\|O M\|<\varepsilon\right\}$, i.e. the union of cylinders whose diameters are $\varepsilon$ and whose axes are the $e_{i}$ segments. The domain $\Omega^{\varepsilon}$ is not smooth at the junctions, but we observe the convergence to the asymptotic problem 1.2 anyway.

Let us take the following boundary conditions: 
Table 3: $\ell^{\infty}$-error on $\frac{\partial P}{\partial x^{(e)}}$ for the disc cross-section, $\beta=1, p(x, t)=\mathrm{e}^{(1-t) x-1 /(t+\varepsilon)}$.

\begin{tabular}{|c|c|c|c|c|c|c|c|c|}
\hline & kernel & & Numerical & & Corrected & & Exact & \\
\hline$h$ & $k$ & $H$ & $\ell^{\infty}$-error $p_{x}$ & order & $\ell^{\infty}$-error $p_{x}$ & order & $\ell^{\infty}$-error $p_{x}$ & order \\
\hline $2^{-1}$ & & & $1.37116 \mathrm{e}-06$ & & $1.37126 \mathrm{e}-06$ & & $1.37063 \mathrm{e}-06$ & \\
\hline $2^{-2}$ & & & $3.98424 \mathrm{e}-07$ & 1.78 & $3.98532 \mathrm{e}-07$ & 1.78 & $3.97835 \mathrm{e}-07$ & 1.78 \\
\hline $2^{-3}$ & & & $1.07928 \mathrm{e}-07$ & 1.88 & $1.08041 \mathrm{e}-07$ & 1.88 & $1.07306 \mathrm{e}-07$ & 1.89 \\
\hline $2^{-4}$ & & & $2.85134 \mathrm{e}-08$ & 1.92 & $2.86301 \mathrm{e}-08$ & 1.92 & $2.78745 \mathrm{e}-08$ & 1.94 \\
\hline $2^{-5}$ & $0.1 \cdot 2^{-14}$ & $2^{-10} \pi$ & $7.75301 \mathrm{e}-09$ & 1.88 & $7.87126 \mathrm{e}-09$ & 1.86 & 7.10518e-09 & 1.97 \\
\hline $2^{-6}$ & & & $2.44720 \mathrm{e}-09$ & 1.66 & $2.56627 \mathrm{e}-09$ & 1.62 & $1.79485 \mathrm{e}-09$ & 1.99 \\
\hline $2^{-7}$ & & & $1.10689 \mathrm{e}-09$ & 1.14 & $1.22637 \mathrm{e}-09$ & 1.07 & $4.52255 \mathrm{e}-10$ & 1.99 \\
\hline $2^{-8}$ & & & $7.70502 \mathrm{e}-10$ & 0.52 & $8.90184 \mathrm{e}-10$ & 0.46 & $1.14715 \mathrm{e}-10$ & 1.98 \\
\hline $2^{-9}$ & & & $6.86454 \mathrm{e}-10$ & 0.17 & $8.06241 \mathrm{e}-10$ & 0.14 & $3.00928 \mathrm{e}-11$ & 1.93 \\
\hline $2^{-10}$ & & & $6.65557 \mathrm{e}-10$ & 0.04 & $7.85396 \mathrm{e}-10$ & 0.04 & $8.90762 \mathrm{e}-12$ & 1.76 \\
\hline \multirow{14}{*}{$2^{-10}$} & $0.1 \cdot 2^{-1}$ & \multirow{14}{*}{$2^{-10} \pi$} & $1.40603 \mathrm{e}-05$ & & $1.50528 \mathrm{e}-05$ & & $1.70035 \mathrm{e}-05$ & \\
\hline & $0.1 \cdot 2^{-2}$ & & $8.23443 \mathrm{e}-06$ & 0.77 & $9.36902 \mathrm{e}-06$ & 0.68 & $1.16079 \mathrm{e}-05$ & 0.55 \\
\hline & $0.1 \cdot 2^{-3}$ & & $1.46155 \mathrm{e}-06$ & 2.49 & $3.60481 \mathrm{e}-06$ & 1.38 & $4.71718 \mathrm{e}-06$ & 1.30 \\
\hline & $0.1 \cdot 2^{-4}$ & & $7.63423 \mathrm{e}-07$ & 0.94 & $1.03297 \mathrm{e}-06$ & 1.80 & $1.49344 \mathrm{e}-06$ & 1.66 \\
\hline & $0.1 \cdot 2^{-5}$ & & $7.67132 \mathrm{e}-07$ & -0.01 & $2.91920 \mathrm{e}-07$ & 1.82 & $4.20353 \mathrm{e}-07$ & 1.83 \\
\hline & $0.1 \cdot 2^{-6}$ & & $4.14515 \mathrm{e}-07$ & 0.89 & $6.10037 \mathrm{e}-08$ & 2.26 & $1.11756 \mathrm{e}-07$ & 1.91 \\
\hline & $0.1 \cdot 2^{-7}$ & & $1.82874 \mathrm{e}-07$ & 1.18 & $1.23173 \mathrm{e}-08$ & 2.31 & $2.88777 \mathrm{e}-08$ & 1.95 \\
\hline & $0.1 \cdot 2^{-8}$ & & $7.31796 \mathrm{e}-08$ & 1.32 & $1.70640 \mathrm{e}-09$ & 2.85 & $7.35660 \mathrm{e}-09$ & 1.97 \\
\hline & $0.1 \cdot 2^{-9}$ & & $2.75420 \mathrm{e}-08$ & 1.41 & $4.54956 \mathrm{e}-10$ & 1.91 & $1.86343 \mathrm{e}-09$ & 1.98 \\
\hline & $0.1 \cdot 2^{-10}$ & & $9.73626 \mathrm{e}-09$ & 1.50 & $4.85544 \mathrm{e}-10$ & -0.09 & $4.73955 \mathrm{e}-10$ & 1.98 \\
\hline & $0.1 \cdot 2^{-11}$ & & $3.03157 \mathrm{e}-09$ & 1.68 & $6.40152 \mathrm{e}-10$ & -0.40 & $1.24211 \mathrm{e}-10$ & 1.93 \\
\hline & $0.1 \cdot 2^{-12}$ & & $5.59160 \mathrm{e}-10$ & 2.44 & $7.17743 \mathrm{e}-10$ & -0.17 & $3.64187 \mathrm{e}-11$ & 1.77 \\
\hline & $0.1 \cdot 2^{-13}$ & & $3.40868 \mathrm{e}-10$ & 0.71 & $7.60524 \mathrm{e}-10$ & -0.08 & $1.44120 \mathrm{e}-11$ & 1.34 \\
\hline & $0.1 \cdot 2^{-14}$ & & $6.65557 \mathrm{e}-10$ & -0.97 & $7.85396 \mathrm{e}-10$ & -0.05 & $8.90762 \mathrm{e}-12$ & 0.69 \\
\hline \multirow{10}{*}{$2^{-10}$} & \multirow{10}{*}{$0.1 \cdot 2^{-14}$} & $2^{-1} \pi$ & $3.69456 \mathrm{e}-05$ & & $3.68546 \mathrm{e}-05$ & & & \\
\hline & & $2^{-2} \pi$ & $3.36543 \mathrm{e}-07$ & 6.78 & $3.85246 \mathrm{e}-07$ & 6.58 & & \\
\hline & & $2^{-3} \pi$ & $2.97071 \mathrm{e}-06$ & -3.14 & $3.01664 \mathrm{e}-06$ & -2.97 & & \\
\hline & & $2^{-4} \pi$ & $7.43567 \mathrm{e}-07$ & 2.00 & $7.86382 \mathrm{e}-07$ & 1.94 & & \\
\hline & & $2^{-5} \pi$ & $8.39831 \mathrm{e}-08$ & 3.15 & $6.60872 \mathrm{e}-08$ & 3.57 & & \\
\hline & & $2^{-6} \pi$ & $9.45994 \mathrm{e}-08$ & -0.17 & $1.13113 \mathrm{e}-07$ & -0.78 & & \\
\hline & & $2^{-7} \pi$ & $3.63271 \mathrm{e}-08$ & 1.38 & $4.33101 \mathrm{e}-08$ & 1.38 & & \\
\hline & & $2^{-8} \pi$ & $1.13907 \mathrm{e}-08$ & 1.67 & $1.22260 \mathrm{e}-08$ & 1.82 & & \\
\hline & & $2^{-9} \pi$ & $3.07604 \mathrm{e}-09$ & 1.89 & $3.17743 \mathrm{e}-09$ & 1.94 & & \\
\hline & & $2^{-10} \pi$ & $6.65557 \mathrm{e}-10$ & 2.21 & $7.85396 \mathrm{e}-10$ & 2.02 & & \\
\hline
\end{tabular}


Table 4: $\ell^{\infty}$-error on $\frac{\partial P}{\partial x^{(e)}}$ for the disc cross-section, $\beta=0, p(x, t)=\mathrm{e}^{(1-t) x}$.

\begin{tabular}{|c|c|c|c|c|c|c|c|c|}
\hline & kernel & & Numerical & & Corrected & & Exact & \\
\hline$h$ & $k$ & $H$ & $\ell^{\infty}$-error $p_{x}$ & order & $\ell^{\infty}$-error $p_{x}$ & order & $\ell^{\infty}$-error $p_{x}$ & order \\
\hline $2^{-1}$ & \multirow{10}{*}{$0.1 \cdot 2^{-14}$} & \multirow{10}{*}{$2^{-10} \pi$} & $4.65502 \mathrm{e}-02$ & & $4.65403 \mathrm{e}-02$ & & $4.64901 \mathrm{e}-02$ & \\
\hline $2^{-2}$ & & & $1.36905 \mathrm{e}-02$ & 1.77 & $1.36707 \mathrm{e}-02$ & 1.77 & $1.36146 \mathrm{e}-02$ & 1.77 \\
\hline $2^{-3}$ & & & $3.77399 \mathrm{e}-03$ & 1.86 & $3.75018 \mathrm{e}-03$ & 1.87 & $3.69076 \mathrm{e}-03$ & 1.88 \\
\hline $2^{-4}$ & & & $2.42701 \mathrm{e}-03$ & 0.64 & $1.02249 \mathrm{e}-03$ & 1.87 & $9.61254 \mathrm{e}-04$ & 1.94 \\
\hline $2^{-5}$ & & & $3.19538 \mathrm{e}-03$ & -0.40 & $3.07484 \mathrm{e}-04$ & 1.73 & $2.45304 \mathrm{e}-04$ & 1.97 \\
\hline $2^{-6}$ & & & $3.40548 \mathrm{e}-03$ & -0.09 & $1.24617 \mathrm{e}-04$ & 1.30 & $6.19574 \mathrm{e}-05$ & 1.99 \\
\hline $2^{-7}$ & & & $3.46538 \mathrm{e}-03$ & -0.03 & $7.84674 \mathrm{e}-05$ & 0.67 & $1.55663 \mathrm{e}-05$ & 1.99 \\
\hline $2^{-8}$ & & & $3.48384 \mathrm{e}-03$ & -0.01 & $6.69214 \mathrm{e}-05$ & 0.23 & $3.89957 \mathrm{e}-06$ & 2.00 \\
\hline $2^{-9}$ & & & $3.49017 \mathrm{e}-03$ & -0.00 & $6.40570 \mathrm{e}-05$ & 0.06 & $9.74829 \mathrm{e}-07$ & 2.00 \\
\hline $2^{-10}$ & & & $3.49261 \mathrm{e}-03$ & -0.00 & $6.33551 \mathrm{e}-05$ & 0.02 & $2.43051 \mathrm{e}-07$ & 2.00 \\
\hline \multirow{14}{*}{$2^{-10}$} & $0.1 \cdot 2^{-1}$ & \multirow{14}{*}{$2^{-10} \pi$} & $3.74748 \mathrm{e}-01$ & & $2.51838 \mathrm{e}-01$ & & $1.02594 \mathrm{e}-02$ & \\
\hline & $0.1 \cdot 2^{-2}$ & & $2.51240 \mathrm{e}-01$ & 0.58 & $1.67151 \mathrm{e}-01$ & 0.59 & $3.50858 \mathrm{e}-03$ & 1.55 \\
\hline & $0.1 \cdot 2^{-3}$ & & $1.70551 \mathrm{e}-01$ & 0.56 & $5.48469 \mathrm{e}-02$ & 1.61 & $1.21113 \mathrm{e}-03$ & 1.53 \\
\hline & $0.1 \cdot 2^{-4}$ & & $1.17002 \mathrm{e}-01$ & 0.54 & $1.84214 \mathrm{e}-02$ & 1.57 & $4.20822 \mathrm{e}-04$ & 1.53 \\
\hline & $0.1 \cdot 2^{-5}$ & & $8.09255 \mathrm{e}-02$ & 0.53 & $4.56308 \mathrm{e}-03$ & 2.01 & $1.46833 \mathrm{e}-04$ & 1.52 \\
\hline & $0.1 \cdot 2^{-6}$ & & $5.63137 \mathrm{e}-02$ & 0.52 & $1.82718 \mathrm{e}-03$ & 1.32 & $5.13093 \mathrm{e}-05$ & 1.52 \\
\hline & $0.1 \cdot 2^{-7}$ & & $3.93576 \mathrm{e}-02$ & 0.52 & $6.16791 \mathrm{e}-04$ & 1.57 & $1.78715 \mathrm{e}-05$ & 1.52 \\
\hline & $0.1 \cdot 2^{-8}$ & & $2.75894 \mathrm{e}-02$ & 0.51 & $2.57248 \mathrm{e}-04$ & 1.26 & $6.13280 \mathrm{e}-06$ & 1.54 \\
\hline & $0.1 \cdot 2^{-9}$ & & $1.93776 \mathrm{e}-02$ & 0.51 & $8.02652 \mathrm{e}-05$ & 1.68 & $2.00335 \mathrm{e}-06$ & 1.61 \\
\hline & $0.1 \cdot 2^{-10}$ & & $1.36270 \mathrm{e}-02$ & 0.51 & $1.50374 \mathrm{e}-05$ & 2.42 & $5.48597 \mathrm{e}-07$ & 1.87 \\
\hline & $0.1 \cdot 2^{-11}$ & & $9.59438 \mathrm{e}-03$ & 0.51 & $3.52991 \mathrm{e}-05$ & -1.23 & $2.20105 \mathrm{e}-07$ & 1.32 \\
\hline & $0.1 \cdot 2^{-12}$ & & $6.77321 \mathrm{e}-03$ & 0.50 & $4.65737 \mathrm{e}-05$ & -0.40 & $2.35100 \mathrm{e}-07$ & -0.10 \\
\hline & $0.1 \cdot 2^{-13}$ & & $4.81817 \mathrm{e}-03$ & 0.49 & $5.50126 \mathrm{e}-05$ & -0.24 & $2.40775 \mathrm{e}-07$ & -0.03 \\
\hline & $0.1 \cdot 2^{-14}$ & & $3.49261 \mathrm{e}-03$ & 0.46 & $6.33551 \mathrm{e}-05$ & -0.20 & $2.43051 \mathrm{e}-07$ & -0.01 \\
\hline \multirow{10}{*}{$2^{-10}$} & \multirow{10}{*}{$0.1 \cdot 2^{-14}$} & $2^{-1} \pi$ & $1.53506 \mathrm{e}+00$ & & $1.37410 \mathrm{e}+00$ & & & \\
\hline & & $2^{-2} \pi$ & $2.89715 \mathrm{e}-01$ & 2.41 & $2.13087 \mathrm{e}-01$ & 2.69 & & \\
\hline & & $2^{-3} \pi$ & $1.26877 \mathrm{e}-01$ & 1.19 & $1.26814 \mathrm{e}-01$ & 0.75 & & \\
\hline & & $2^{-4} \pi$ & $7.56310 \mathrm{e}-02$ & 0.75 & $7.59343 \mathrm{e}-02$ & 0.74 & & \\
\hline & & $2^{-5} \pi$ & $4.20970 \mathrm{e}-02$ & 0.85 & $5.09920 \mathrm{e}-02$ & 0.57 & & \\
\hline & & $2^{-6} \pi$ & $2.21178 \mathrm{e}-02$ & 0.93 & $8.15857 \mathrm{e}-03$ & 2.64 & & \\
\hline & & $2^{-7} \pi$ & $1.14523 \mathrm{e}-02$ & 0.95 & $1.85572 \mathrm{e}-03$ & 2.14 & & \\
\hline & & $2^{-8} \pi$ & $6.01148 \mathrm{e}-03$ & 0.93 & $8.35024 \mathrm{e}-04$ & 1.15 & & \\
\hline & & $2^{-9} \pi$ & $4.15333 \mathrm{e}-03$ & 0.53 & $2.39586 \mathrm{e}-04$ & 1.80 & & \\
\hline & & $2^{-10} \pi$ & $3.49261 \mathrm{e}-03$ & 0.25 & $6.33551 \mathrm{e}-05$ & 1.92 & & \\
\hline
\end{tabular}



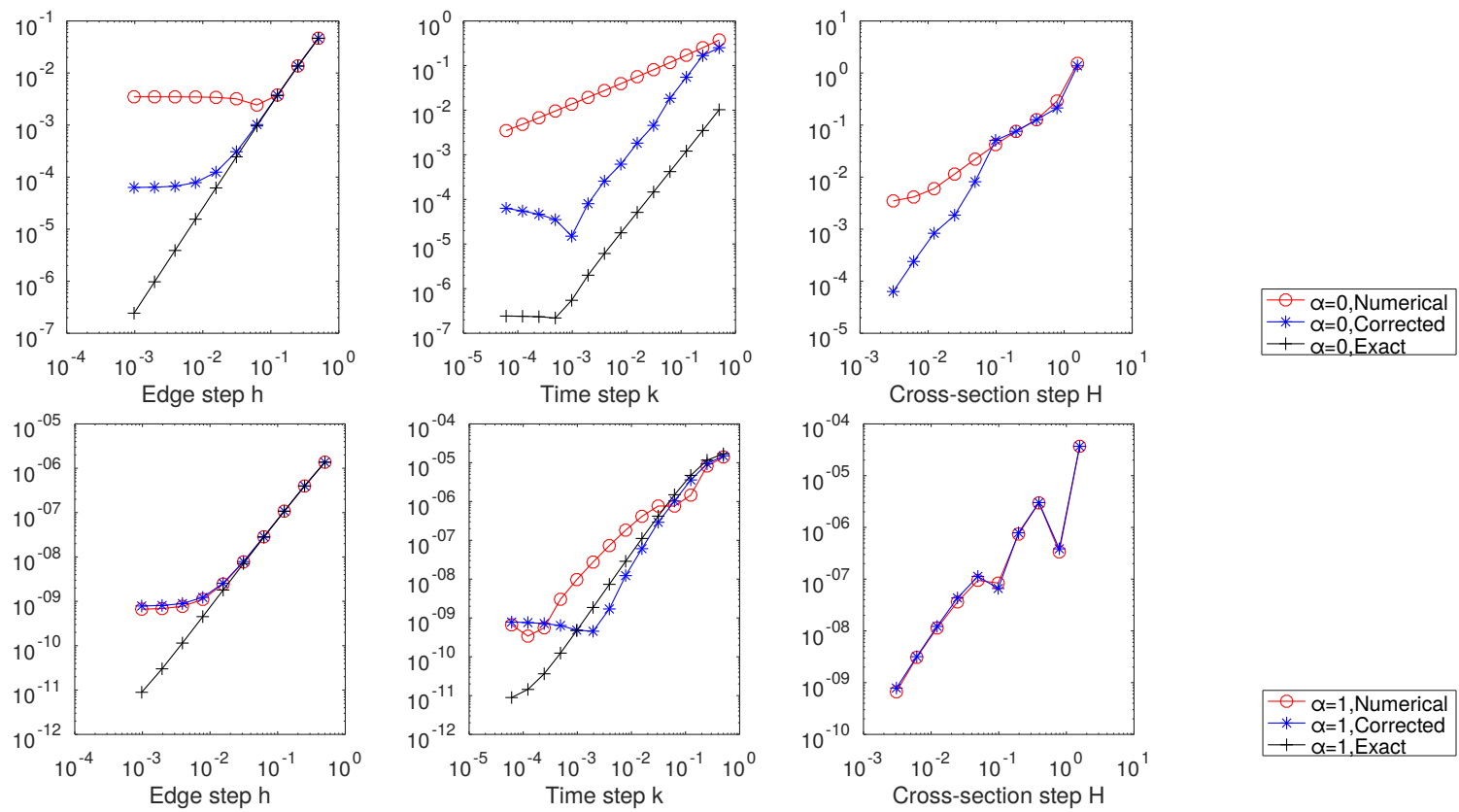

$+\alpha=0$, Exact

Figure 2: $\quad \ell^{\infty}$-error on $\frac{\partial P}{\partial x^{(e)}}$ curves obtained from the data presented in Table 4 (top: $\beta=0$ ) and Table 3 (bottom: $\beta=1$ ). On each graph, only one parameter varies, the two others are set by default to $h=2^{-10}, k=0.1 \cdot 2^{-14}, H=\pi 2^{-10}$.

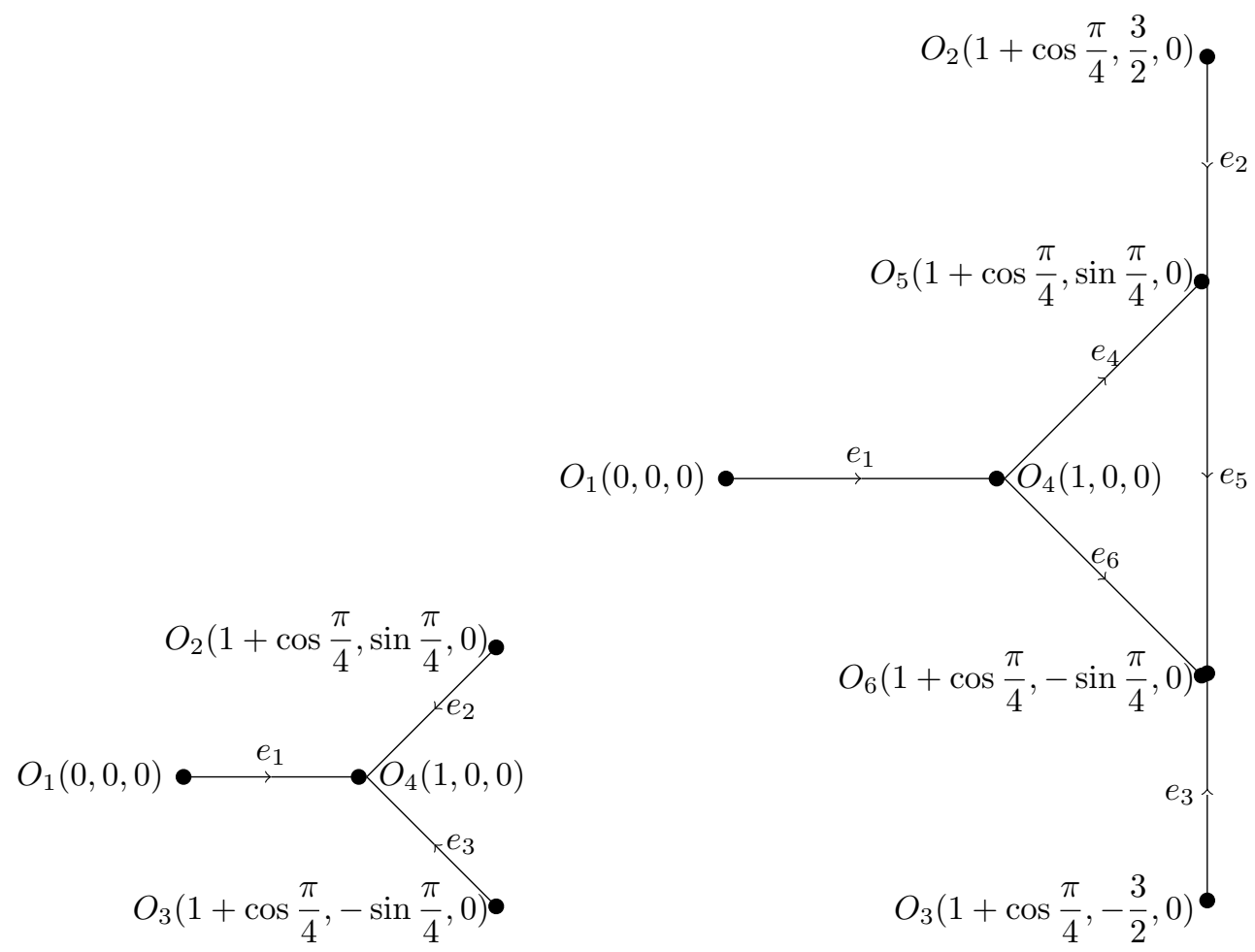

Figure 3: The full dimension Navier-Stokes simulations were run on two geometries. On the left, the simply connected one $\left(N_{1}=3, N=4, M=3\right)$, and on the right, the multiply connected one $\left(N_{1}=3, N=6, M=6\right)$. 
- $p=0$ and $v(M, t)$ colinear to $e_{1}$ at the beginning $O_{1}$ of $e_{1}$.

- $v(M, t)=-\frac{e_{2}}{\left|e_{2}\right|} v_{0} \sin \left(\varepsilon^{-2} 40 t\right)\left(1-4 \varepsilon^{-2}\left\|O_{2} M\right\|^{2}\right)$ at the beginning $O_{2}$ of $e_{2}$. The flux through this tube is then $\frac{1}{8} \pi v_{0} \varepsilon^{2} \sin \left(\varepsilon^{-2} 40 t\right)$;

- $v(M, t)=2 \frac{e_{3}}{\left|e_{3}\right|} v_{0} \sin \left(\varepsilon^{-2} 40 t\right)\left(1-4 \varepsilon^{-2}\left\|O_{3} M\right\|^{2}\right)$ at the beginning $O_{3}$ of $e_{3}$. The flux through this tube is then $\frac{\pi}{4} v_{0} \varepsilon^{2} \sin \left(\varepsilon^{-2} 40 t\right)$;

- $v=0$ on the rest of the boundary.

Using the mass conservation law, we conclude that the flux through this first tube is: $\frac{3}{8} \pi v_{0} \varepsilon^{2} \sin \left(\varepsilon^{-2} 40 t\right)$.

\subsubsection{Results}

Simulations with COMSOL software run over the interval $\left[0,0.0875 \varepsilon^{2}\right]$ for $\varepsilon \in\{0.2,0.1,0.05,0.025\}$. Let $q^{\varepsilon}$ be the continuous in space function, affine over each edge, such that $q^{\varepsilon}\left(O_{\ell}\right)=p^{\varepsilon}\left(O_{\ell}\right)$ for $\ell \in\{1, \ldots, N\}$

We compute the $L^{2}\left(0, T, H^{1}(\mathcal{B})\right)$-distance between $p^{\varepsilon}$ restricted to the graph, $q^{\varepsilon}$ obtained with these simulations and the pressure $P$ predicted by the asymptotic model. The results are reported in Tables 5 and 6 .

We also show in Figures 4, 5, 6 and 7 the velocity magnitude and the pressure inside $\Omega^{\varepsilon}$. In the figures 6 and 7 . the velocity for the asymptotic problem is obtained using the scheme proposed in Section 3.5 .3 .

Notice that that the velocity magnitude is not convex in the cross-section, unlike the stationary Poiseuille profile.

According to [21, the junction boundary layers have a space width of order of $\varepsilon$. Thus, the order of convergence of $p^{\varepsilon}-q^{\varepsilon}, p^{\varepsilon}-P$ in $L^{2}\left(0, T, H^{1}(\mathcal{B})\right)$ norm is $1 / 2$. The order of convergence of $q^{\varepsilon}-P$ in $L^{2}\left(0, T, H^{1}(\mathcal{B})\right)$ norm is 1 .

This is compatible with what we observe. However, we are limited by the accuracy of the numerical resolution of the Navier-Stokes equations. Anyway, the asymptotic model shows a good agreement with this numerical solution, with only $6 \%$ error when $\varepsilon=0.025$ in the multiply connected geometry case and $1 \%$ error when $\varepsilon \leq 0.1$ in the simply connected geometry case.

We also run simulations in the planar case, with a cut of the multiply connected geometry. This enabled us to use a much smaller space-step and thus to reach a better precision. We take a cut of the multiply connected geometry in the $x y$-plane and run a simulation in the interval $\left[0,0.25 \varepsilon^{2}\right]$ with the Dirichlet boundary conditions for the velocity at the extremities of the three tubes. We use a characteristic Galerkin method, with $\mathbb{P}^{3}-\mathbb{P}^{2}$ Taylor-Hood elements in space and BDF2 time integrator (scheme (12) in [2]). It was implemented with FreeFem ++ [13]. Results are reported on table 7 and match the theoretical results of [21].

For this planar case, we also computed $\tilde{q}_{\varepsilon}$ the function (not necessarily continuous) affine on each edge such $\left(\tilde{q}_{\varepsilon}-p_{\varepsilon}\right)\left(\frac{2}{3} O_{i_{j}}+\frac{1}{3} O_{k_{j}}\right)=0,\left(\tilde{q}_{\varepsilon}-p_{\varepsilon}\right)\left(\frac{1}{3} O_{i_{j}}+\frac{2}{3} O_{k_{j}}\right)=0$ for $j \in\{1, \ldots, M\}$. We observed $\tilde{q}_{\varepsilon}$ converges very rapidly to $P$ on the edges where the flux is prescribed (i.e. $e_{1}, e_{2}, e_{3}$ ) in $L^{2}\left(0, T, H_{d c}^{1}\left(e_{1} \cup e_{2} \cup e_{3}\right)\right)$ norm, with a $0.003 \%$ relative error when $\varepsilon=0.1$. According to [21], the convergence should be exponential. 


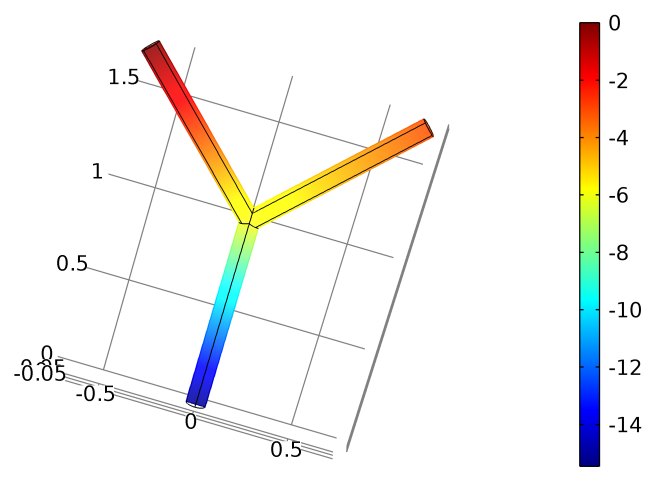

Pressure

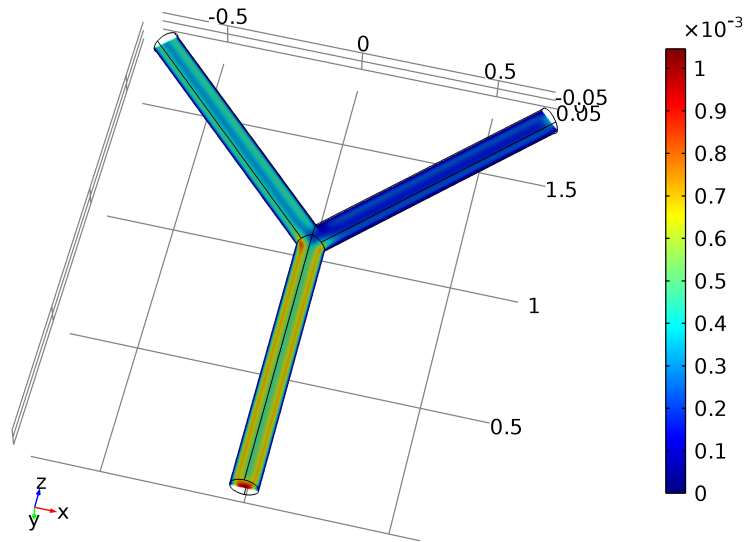

Velocity magnitude

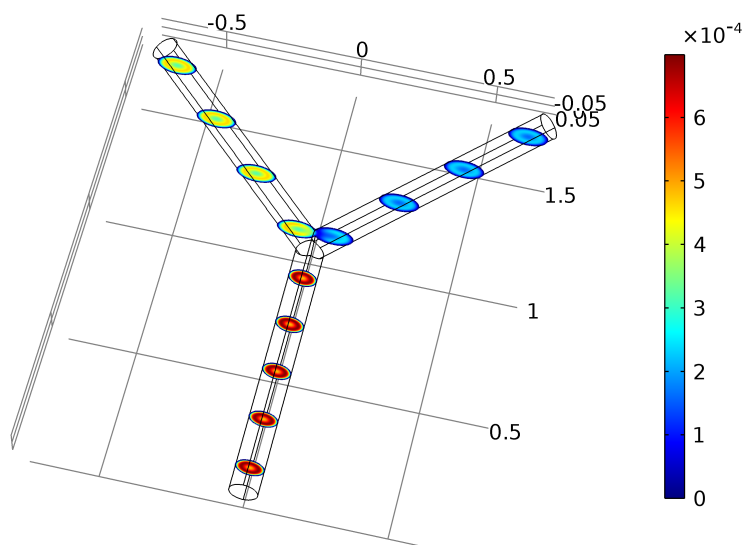

Velocity magnitude

Figure 4: Pressure and velocity magnitude in the transversal and longitudinal cuts of the simply connected geometry at $t=0.0875 \varepsilon^{2}, \varepsilon=0.1$. 


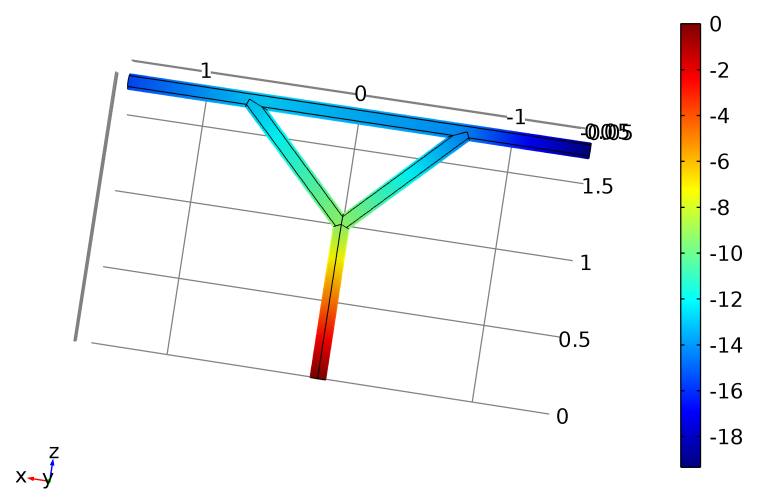

Pressure

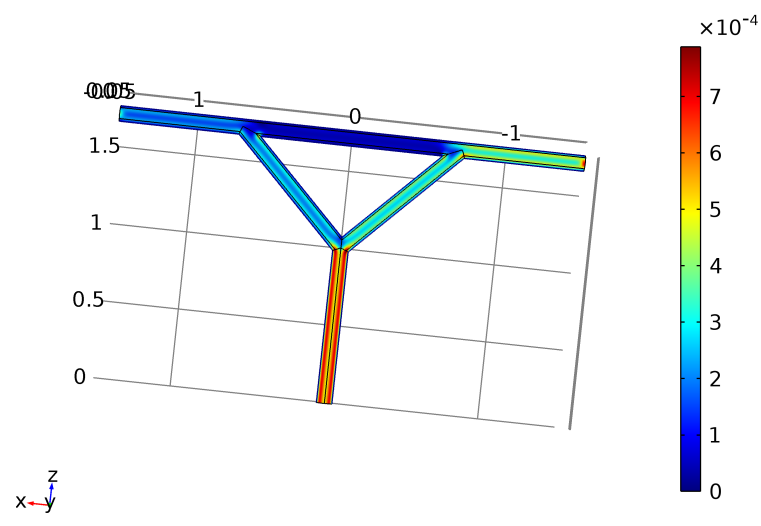

Velocity magnitude

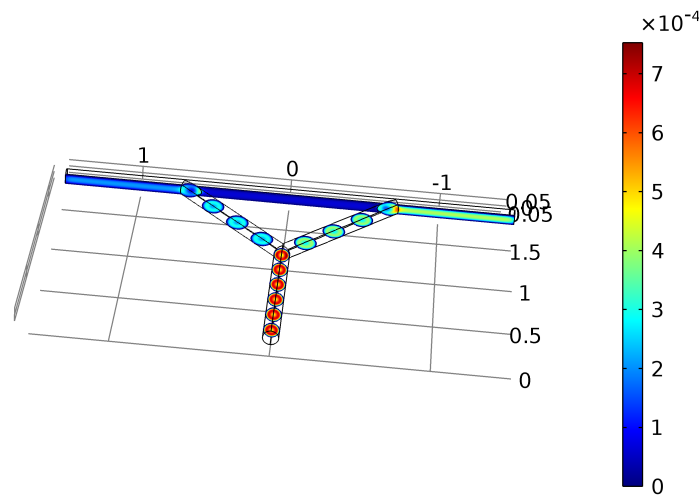

Velocity magnitude

Figure 5: Pressure and velocity magnitude in the transversal and longitudinal cuts of the multiply connected geometry at $t=0.0875 \varepsilon^{2}, \varepsilon=0.1$. 

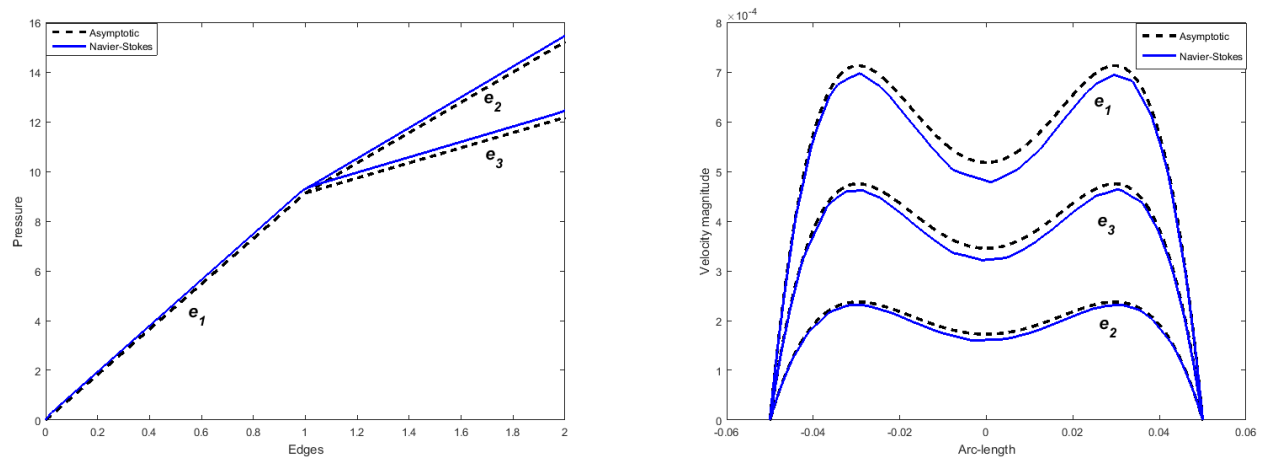

Figure 6: Comparison between the asymptotic model (dashed lines) and the Navier-Stokes numerical solution (blue lines) for the simply connected geometry when $T=0.875 \varepsilon^{2}, \varepsilon=0.1$. On the left, the pressure along tubes. On the right, the velocity magnitude across the middle of the three tubes with respect to the distance to the axis of the tube
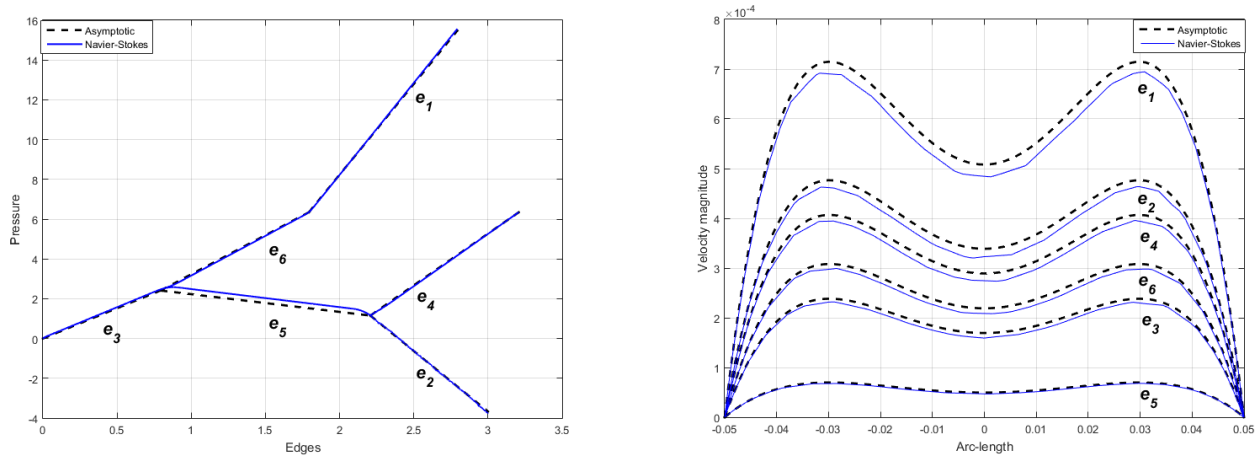

Figure 7: Comparison between the asymptotic model (dashed lines) and the Navier-Stokes numerical solution (blue lines) for the multiply connected geometry when $T=0.0875 \varepsilon^{2}, \varepsilon=0.1$. On the left, the pressure along tubes. On the right, the velocity magnitude across the middle of the six tubes with respect to the distance to the axis of the tube

Table 5: Comparison between Navier-Stokes numerical solution and the asymptotic model in the case of the simply connected geometry. $T=0.875 \varepsilon^{2}$. In this table, $\Phi_{j}^{\varepsilon}, \Phi_{j}$ is the flux accross the $j$-th tube according to Navier-Stokes numerical solution and the asymptotic model. The last line of the table is an estimate of the accuracy of the numerical method used to compute the Navier-Stokes solution.

\begin{tabular}{lcccc}
\hline$\frac{\left\|P-p^{\varepsilon}\right\|_{L^{2}\left(0, T, H^{1}(\mathcal{B})\right)}}{\left\|p^{\varepsilon}\right\|_{L^{2}\left(0, T, H^{1}(\mathcal{B})\right)}}$ & 0.2 & 0.1 & 0.05 & 0.025 \\
\hline$\frac{\left\|p^{\varepsilon}-q^{\varepsilon}\right\|_{L^{2}\left(0, T, H^{1}(\mathcal{B})\right)}}{\left\|p^{\varepsilon}\right\|_{L^{2}\left(0, T, H^{1}(\mathcal{B})\right)}}$ & 0.0968455 & 0.07037690 .46 & 0.05329790 .40 & 0.04185590 .35 \\
\hline$\frac{\left\|P-q^{\varepsilon}\right\|_{L^{2}\left(0, T, H^{1}(\mathcal{B})\right)}}{\left\|q^{\varepsilon}\right\|_{L^{2}\left(0, T, H^{1}(\mathcal{B})\right)}}$ & 0.0940223 & 0.06888500 .45 & 0.05248120 .39 & 0.04026380 .38 \\
\hline$\frac{\left\|\left(\Phi_{j}-\Phi_{j}^{\varepsilon}\right)_{j}\right\|_{L^{2}(\{1, \ldots, M\} \times[0, T])}}{\left\|\left(\Phi_{j}^{\varepsilon}\right)_{j}\right\|_{L^{2}(\{1, \ldots, M\} \times[0, T])}}$ & 0.0233166 & 0.01444850 .69 & 0.00930760 .63 & $0.0114437-.30$ \\
\hline
\end{tabular}


Table 6: Comparison between Navier-Stokes numerical solution and the asymptotic model in the case of the multiply-connected geometry. $T=0.875 \varepsilon^{2}$. In this table, $\Phi_{j}^{\varepsilon}, \Phi_{j}$ is the flux accross the $j$-th tube according to Navier-Stokes numerical solution and the asymptotic model, and $\Psi_{\ell}^{\varepsilon}=$ $\sum_{O_{\ell} \in e_{j}} \alpha_{\ell, j} \Phi_{j}^{\varepsilon}$. The last line of the table is an estimate of the accuracy of the numerical method used to compute the Navier-Stokes solution.

\begin{tabular}{lcccc}
\hline$\varepsilon$ & 0.2 & 0.1 & 0.05 & 0.025 \\
\hline$\frac{\left\|P-p^{\varepsilon}\right\|_{L^{2}\left(0, T, H^{1}(\mathcal{B})\right)}}{\left\|p^{\varepsilon}\right\|_{L^{2}\left(0, T, H^{1}(\mathcal{B})\right)}}$ & 0.144626 & 0.1035210 .48 & 0.0800280 .37 & 0.0627300 .35 \\
\hline$\frac{\left\|p^{\varepsilon}-q^{\varepsilon}\right\|_{L^{2}\left(0, T, H^{1}(\mathcal{B})\right)}}{\left\|p^{\varepsilon}\right\|_{L^{2}\left(0, T, H^{1}(\mathcal{B})\right)}}$ & 0.140002 & 0.1013880 .47 & 0.0735400 .46 & 0.0561460 .39 \\
\hline$\frac{\left\|P-q^{\varepsilon}\right\|_{L^{2}\left(0, T, H^{1}(\mathcal{B})\right)}}{\left\|q^{\varepsilon}\right\|_{L^{2}\left(0, T, H^{1}(\mathcal{B})\right)}}$ & 0.036639 & 0.0210160 .80 & $0.031650-.59$ & 0.0280220 .18 \\
\hline$\frac{\left\|\left(\Phi_{j}-\Phi_{j}^{\varepsilon}\right)_{j}\right\|_{L^{2}(\{1, \ldots, M\} \times[0, T])}}{\left\|\left(\Phi_{j}^{\varepsilon}\right)_{j}\right\|_{L^{2}(\{1, \ldots, M\} \times[0, T])}}$ & 0.035878 & 0.0301960 .25 & $0.056603-.91$ & 0.0536610 .08 \\
$\frac{\left\|\left(\Psi_{\ell}-\Psi_{\ell}^{\varepsilon}\right)_{\ell}\right\|_{L^{2}(\{1, \ldots, N\} \times[0, T])}}{\left\|\left(\Psi_{\ell}^{\varepsilon}\right)_{\ell}\right\|_{L^{2}(\{1, \ldots, N\} \times[0, T])}}$ & 0.035841 & 0.0301090 .25 & $0.056649-.91$ & 0.0532750 .09 \\
\hline
\end{tabular}

Table 7: Multiply connected two-dimensional case. $\Phi_{j}^{\varepsilon}, \Phi_{j}, \Psi_{j}^{\varepsilon}$ denotes the same fluxes as in Table 6 . Notice in column 5 that $\tilde{q}_{\varepsilon}$ is very accurately predicted by $P$ on edges $(j \in\{1,2,3\})$ on which the flux is imposed.

\begin{tabular}{|c|c|c|c|}
\hline \multirow{2}{*}{$\varepsilon$} & $\left\|P-p^{\varepsilon}\right\|_{L^{2}\left(0, T, H^{1}(\mathcal{B})\right)}$ & $\left\|p^{\varepsilon}-q^{\varepsilon}\right\|_{L^{2}\left(0, T, H^{1}(\mathcal{B})\right)}$ & $\left\|P-q^{\varepsilon}\right\|_{L^{2}\left(0, T, H^{1}(\mathcal{B})\right)}$ \\
\hline & $\left\|p^{\varepsilon}\right\|_{L^{2}\left(0, T, H^{1}(\mathcal{B})\right)}$ & $\left\|p^{\varepsilon}\right\|_{L^{2}\left(0, T, H^{1}(\mathcal{B})\right)}$ & $\left\|q^{\varepsilon}\right\|_{L^{2}\left(0, T, H^{1}(\mathcal{B})\right)}$ \\
\hline $4 \mathrm{e}-01$ & $1.84158 \mathrm{e}-01$ & $1.74262 \mathrm{e}-01$ & $6.04826 \mathrm{e}-02$ \\
\hline $3 e-01$ & $1.61135 \mathrm{e}-010.46$ & $1.54638 \mathrm{e}-010.42$ & $4.58469 \mathrm{e}-020.96$ \\
\hline $2 \mathrm{e}-01$ & $1.33035 \mathrm{e}-010.47$ & $1.29469 \mathrm{e}-010.44$ & $3.08584 \mathrm{e}-020.98$ \\
\hline $1 \mathrm{e}-01$ & $9.51649 \mathrm{e}-02 \quad 0.48$ & $9.38936 \mathrm{e}-02 \quad 0.46$ & $1.55718 \mathrm{e}-020.99$ \\
\hline $7 \mathrm{e}-02$ & 7.99077e-02 0.49 & 7.91613e-02 0.48 & $1.09305 \mathrm{e}-020.99$ \\
\hline $5 e-02$ & $6.76983 \mathrm{e}-020.49$ & $6.72472 \mathrm{e}-020.48$ & 7.82038e-03 1.00 \\
\hline $4 e-02$ & $6.06171 \mathrm{e}-020.50$ & $6.02939 \mathrm{e}-020.49$ & $6.26215 \mathrm{e}-031.00$ \\
\hline $3 e-02$ & $5.25627 \mathrm{e}-020.50$ & $5.23528 \mathrm{e}-020.49$ & $4.70006 \mathrm{e}-031.00$ \\
\hline $2 \mathrm{e}-02$ & $4.29754 \mathrm{e}-02 \quad 0.50$ & $4.28610 \mathrm{e}-020.49$ & $3.13686 \mathrm{e}-031.00$ \\
\hline $7 \mathrm{e}-03$ & $2.54107 \mathrm{e}-020.50$ & $2.53871 \mathrm{e}-020.50$ & $1.09576 \mathrm{e}-031.00$ \\
\hline $5 \mathrm{e}-03$ & $2.15416 \mathrm{e}-020.49$ & $2.15236 \mathrm{e}-020.49$ & $8.81800 \mathrm{e}-040.65$ \\
\hline \multirow{2}{*}{$\varepsilon$} & $\left\|\left(\Phi_{j}-\Phi_{j}^{\varepsilon}\right)_{j}\right\|_{L^{2}(\{1, \ldots, M\} \times[0, T])}$ & $\left\|P-\tilde{q}^{\varepsilon}\right\|_{L^{2}\left(0, T, H_{d c}^{1}\left(e_{1} \cup e_{2} \cup e_{3}\right)\right)}$ & $\left\|\left(\Psi_{\ell}-\Psi_{\ell}^{\varepsilon}\right)_{\ell}\right\|_{L^{2}(\{1, \ldots, N\} \times[0, T])}$ \\
\hline & $\left\|\left(\Phi_{j}^{\varepsilon}\right)_{j}\right\|_{L^{2}(\{1, \ldots, M\} \times[0, T])}$ & $\left\|\tilde{q}^{\varepsilon}\right\|_{L^{2}\left(0, T, H_{d c}^{1}\left(e_{1} \cup e_{2} \cup e_{3}\right)\right)}$ & $\left\|\left(\Psi_{\ell}^{\varepsilon}\right)_{\ell}\right\|_{L^{2}(\{1, \ldots, N\} \times[0, T])}$ \\
\hline $4 \mathrm{e}-01$ & $1.61063 \mathrm{e}-02$ & $1.99512 \mathrm{e}-04$ & $1.06522 \mathrm{e}-06$ \\
\hline $3 e-01$ & $1.04089 \mathrm{e}-021.52$ & $3.58527 \mathrm{e}-055.97$ & $4.56238 \mathrm{e}-09$ \\
\hline $2 \mathrm{e}-01$ & 6.08976e-03 1.32 & $3.34245 \mathrm{e}-065.85$ & $8.20514 \mathrm{e}-09$ \\
\hline $1 \mathrm{e}-01$ & $2.71136 \mathrm{e}-031.17$ & $3.20877 \mathrm{e}-060.06$ & $2.40961 \mathrm{e}-08$ \\
\hline $7 \mathrm{e}-02$ & $1.84568 \mathrm{e}-031.08$ & $4.59828 \mathrm{e}-06-1.01$ & $4.19010 \mathrm{e}-08$ \\
\hline $5 \mathrm{e}-02$ & $1.28786 \mathrm{e}-031.07$ & $8.35613 \mathrm{e}-06-1.78$ & $1.63812 \mathrm{e}-07$ \\
\hline $4 \mathrm{e}-02$ & $1.02498 \mathrm{e}-031.02$ & $1.21299 \mathrm{e}-05-1.67$ & $2.76485 \mathrm{e}-07$ \\
\hline $3 \mathrm{e}-02$ & $7.58649 \mathrm{e}-041.05$ & $1.71698 \mathrm{e}-05-1.21$ & $3.57710 \mathrm{e}-07$ \\
\hline $2 \mathrm{e}-02$ & 5.01930e-04 1.02 & $2.57071 \mathrm{e}-05-1.00$ & $8.34215 \mathrm{e}-07$ \\
\hline $7 \mathrm{e}-03$ & $1.74694 \mathrm{e}-041.01$ & $8.98785 \mathrm{e}-05-1.19$ & $1.82707 \mathrm{e}-05$ \\
\hline $5 \mathrm{e}-03$ & $1.37612 \mathrm{e}-040.71$ & $3.15042 \mathrm{e}-04-3.73$ & $6.95978 \mathrm{e}-05$ \\
\hline
\end{tabular}




\section{Acknowledgements}

The authors are grateful to Konstantin Pileckas (University of Vilnius) for useful discussions. This work was partially supported by the European Social Fund according to the activity "Improvement of researchers qualification by implementing world-class R and D projects" No. 09.3.3-LMT-K-712.

\section{References}

[1] Igor Ashmetkov, Sergei Mukhin, Nikolai Sosnin, and Anton Favorskii. A boundary value problem for the linearized haemodynamic equations on a graph. Differ. Equations, 40(1):94$104,2004$.

[2] Rodolfo Bermejo and Laura Saavedra. Lagrange-Galerkin methods for the incompressible Navier-Stokes equations: a review. Communications in Applied and Industrial Mathematics, 7.3 (2016): $26-55$.

[3] Cristóbal Bertoglio and Alfonso Caiazzo. A Stokes-residual backflow stabilization method applied to physiological flows. Journal of Computational Physics, 313:260-278, may 2016.

[4] Cristóbal Bertoglio, Alfonso Caiazzo, Yuri Bazilevs, Malte Braack, Mahdi Esmaily, Volker Gravemeier, Alison L. Marsden, Olivier Pironneau, Irene E. Vignon-Clementel, and Wolfgang A. Wall. Benchmark problems for numerical treatment of backflow at open boundaries. International Journal for Numerical Methods in Biomedical Engineering, page e2918, sep 2017.

[5] Cristóbal Bertoglio, Alfonso Caiazzo, and Miguel A. Fernández. Fractional-step schemes for the coupling of distributed and lumped models in hemodynamics. SIAM Journal on Scientific Computing, 35(3):B551-B575, jan 2013.

[6] Artyom Borzov, Sergei Mukhin, Nicolai Sosnin. Conservative algorithm of substance transport over a closed graph of cardiovascular system. Russian Journal of Numerical Analysis and Mathematical Modelling, 25(5):413-429, 2012.

[7] Jean Boussinesq. Essai sur la théorie des eaux courantes. Mémoires présentés par divers savants l'académie des sciences de l'institut de France. Impr. nationale, 1877.

[8] Éric Canon, Frédéric Chardard, Grigory Panasenko, Olga S̆tikonienè. Numerical solution of the viscous flows in a network of thin tubes: equations on the cross-section. to appear.

[9] Luca Formaggia, Daniele Lamponi, Alfio Quarteroni. One dimensional models for blood flow in arteries. J. Engrg. Math, 47:251-276, 2003.

[10] Giovanni Paolo Galdi, Konstantin Pileckas, and Ana Leonor Silvestre. On the unsteady Poiseuille flow in a pipe. Zeitschrift für angewandte Mathematik und Physik, 58(6):994-1007, Nov 2007.

[11] Timur Gamilov, Philippe Kopylov, Roman Pryamonosov, Sergey Simakov. Virtual fractional flow reserve assessment in patient-specific coronary networks by $1 \mathrm{D}$ hemodynamic model. Mathematical Models and Methods in Applied Sciences, 30(5):269-276, 2015.

[12] Gung-Min Gie, Chang-Yeol Jung, and Roger Temam. Recent progresses in boundary layer theory. Discrete Contin. Dyn. Syst., 36(5):2521-2583, 2016.

[13] Frédéric Hecht. New developments in FreeFem++. Journal of Numerical Mathematics, 2012, 20, 3-4, 251-265.

[14] William McLean and Vidar Thome Numerical solution of an evolution equation with a positivetype memory term J. Austral. Math. Soc. Ser. B, 35, 23-70, 1993.

[15] Mette S Olufsen, Charles S Peskin, Won Yong Kim, Erik M Pedersen, Ali Nadim, and Jesper Larsen. Numerical simulation and experimental validation of blood flow in arteries with structured-tree outflow conditions. Annals of Biomedical Engineering, 28(11):1281-1299, 2000. 
[16] Grigory Panasenko. Partial asymptotic decomposition of domain: Navier-Stokes equation in tube structure. Comptes Rendus de l'Académie des Sciences-Series IIB-Mechanics-PhysicsAstronomy, 326(12):893-898, 1998.

[17] Grigory Panasenko. Asymptotic expansion of the solution of Navier-Stokes equation in tube structure and partial asymptotic decomposition of the domain. Applicable Analysis, 76(34):363-381, 2000.

[18] Grigory Panasenko and Konstantin Pileckas. Asymptotic analysis of the nonsteady viscous flow with a given flow rate in a thin pipe. Appl. Anal., 91(3):559-574, 2012.

[19] Grigory Panasenko and Konstantin Pileckas. Flows in a tube structure: equation on the graph. J. Math. Phys., 55(8):081505, 11, 2014.

[20] Grigory Panasenko and Konstantin Pileckas. Asymptotic analysis of the non-steady NavierStokes equations in a tube structure. I. The case without boundary-layer-in-time. Nonlinear Anal., 122:125-168, 2015.

[21] Grigory Panasenko and Konstantin Pileckas. Asymptotic analysis of the non-steady NavierStokes equations in a tube structure. II. General case. Nonlinear Anal., 125:582-607, 2015.

[22] Grigory Panasenko and Konstantin Pileckas. Divergence equation in thin-tube structures. Appl. Anal., 94(7):1450-1459, 2015.

[23] Konstantin Pileckas. Navier-Stokes system in domains with cylindrical outlets to infinity. Lerays problem. In Handbook of mathematical fluid dynamics, volume 4, chapter 8, pages 445-647. Elsevier, 2007.

[24] Konstantin Pileckas and Vaidas Keblikas. Existence of a nonstationary Poiseuille solution. Siberian Mathematical Journal, 46(3):514-526, May 2005.

[25] Jean Louis Marie Poiseuille. Recherches sur les causes du mouvement du sang dans les vaisseaux capillaires. Impr. royale, 1839.

[26] Muhammad Umar Qureshi, Gareth DA Vaughan, Christopher Sainsbury, Martin Johnson, Charles S Peskin, Mette S Olufsen, and NA Hill. Numerical simulation of blood flow and pressure drop in the pulmonary arterial and venous circulation. Biomechanics and modeling in mechanobiology, 13(5):1137-1154, 2014.

[27] Muhammad-Umar Qureshi, Mitchel Colebank, L.Mihaela Paun, Naomi Chesler, Mansoor A. Haider, Nicholas A. Hill, Dirk Husmeier, Mette Olufsen. A computational study of pulmonary hemodynamics in healthy and hypoxic mice. Preprint arxiv:1712.01699 (2017). https://arxiv.org/abs/1712.01699

[28] Anne M. Robertson, Adelia Sequeira. A director theory approach for modeling blood flow in the arterial system: An alternative to classical 1D models. Mathematical Models and Methods in Applied Sciences, 15(6):871-906, 2005.

[29] Spencer J. Sherwin, Luca Formaggia, Joaquim Peiro. Computational modelling of 1D blood flow with variable mechanical properties and its application to the simulation of wave propagation in the human arterial system. Int J Numer Meth Fl, 43(6-7):673-700, 2003.

[30] Vidar Thomée. Galerkin finite element methods for parabolic problems, volume 25 of Springer Series in Computational Mathematics. Springer-Verlag, Berlin, second edition, 2006.

[31] Yuri Vassilevski, Sergey Simakov, Victoria Salamatova, Yuri Ivanov, Tatiana Dobroserdova. Numerical issues of modelling blood flow in networks of vessels with pathologies. Mathematical Models and Methods in Applied Sciences, 26(6):605-622, 2012.

[32] John R Womersley. Method for the calculation of velocity, rate of flow and viscous drag in arteries when the pressure gradient is known. The Journal of physiology, 127(3):553-563, 1955. 